\title{
Nonlinear Vibration Analysis for Stiffened Cylindrical Shells Subjected to Electromagnetic Environment
}

\author{
Xue-Qin Li $\mathbb{D}^{1},{ }^{1}$ Guang-Chen Bai, ${ }^{1}$ Lu-Kai Song $\mathbb{D}^{2},{ }^{2}$ and Wei Zhang ${ }^{3}$ \\ ${ }^{1}$ School of Energy and Power Engineering, Beihang University, Beijing 100191, China \\ ${ }^{2}$ Research Institute of Aero-Engine, Beihang University, Beijing 100191, China \\ ${ }^{3}$ College of Mechanical Engineering, Beijing University of Technology, Beijing 100124, China
}

Correspondence should be addressed to Lu-Kai Song; songlukai29@163.com

Received 23 March 2021; Accepted 7 July 2021; Published 20 July 2021

Academic Editor: Wei He

Copyright (c) 2021 Xue-Qin Li et al. This is an open access article distributed under the Creative Commons Attribution License, which permits unrestricted use, distribution, and reproduction in any medium, provided the original work is properly cited.

The nonlinear vibration behaviors of stiffened cylindrical shells under electromagnetic excitations, transverse excitations, and inplane excitations are studied for the first time in this paper. Given the first-order shear deformation theory and Hamilton principle, the nonlinear partial differential governing equations of motion are derived with considering the von Karman geometric nonlinearity. By employing the Galerkin discretization procedure, the partial differential equations are diverted to a set of coupled nonlinear ordinary differential equations of motion. Based on the case of $1: 2$ internal resonance and principal resonance-1/2 subharmonic parametric resonance, the multiscale method of perturbation analysis is employed to precisely acquire the fourdimensional nonlinear averaged equations. From the resonant response analysis and nonlinear dynamic simulation, we discovered that the unstable regions of stiffened cylindrical shells can be narrowed by decreasing the external excitation or increasing the magnetic intensity, and their working frequency range can be expanded by reducing the in-plane excitation. Moreover, the different nonlinear dynamic responses of the stiffened cylindrical shell are acquired by controlling stiffener number, stiffener size, and aspect ratio. The presented approach in this paper can provide an efficient analytical framework for nonlinear dynamics theories of stiffened cylindrical shells and will shed light on complex structure design in vibration test engineering.

\section{Introduction}

With the remarkable development of astronautic science and technology (i.e., space station, spacecraft, satellite, and rocket), the advanced and large-thrust electrodynamic vibration shakers are needed to simulate the actual working conditions and test the structural vibration performance [1-3]. As the critical component of electrodynamic vibration shakers, armature structures can generate the wanted vibration waveforms with given vibration signals, and its resonance frequency directly determines the upper limit of the working frequency of vibration shaker $[4,5]$. During operations of vibration shaker, due to the large deformation and structural torsion induced by electromagnetic environment, unstable nonlinear dynamic behaviors such as bifurcation and chaos may appear in armature structure, which will produce vibration waveform deviations and considerably reduce the test accuracy. Therefore, effective nonlinear vibration analysis for armature structures to satisfy the test accuracy becomes increasingly important in space engineering. However, for armature structures operating in an electromagnetic environment (as shown in Figure 1), complex geometric configurations not only exist but also are subjected to transverse excitations, in-plane excitations, and electromagnetic excitations [6-8]. These factors significantly increase the analysis complexity of nonlinear vibrations for armature structures. Up to now, few studies have been performed on revealing the nonlinear vibrations of armature structures under electromagnetic environment, which greatly limits the development of highaccuracy vibration test equipment. The realistic demands on improving vibration tests motivate us to start this research on the nonlinear vibrations of armature structures. In this study, by considering the geometric configurations and 


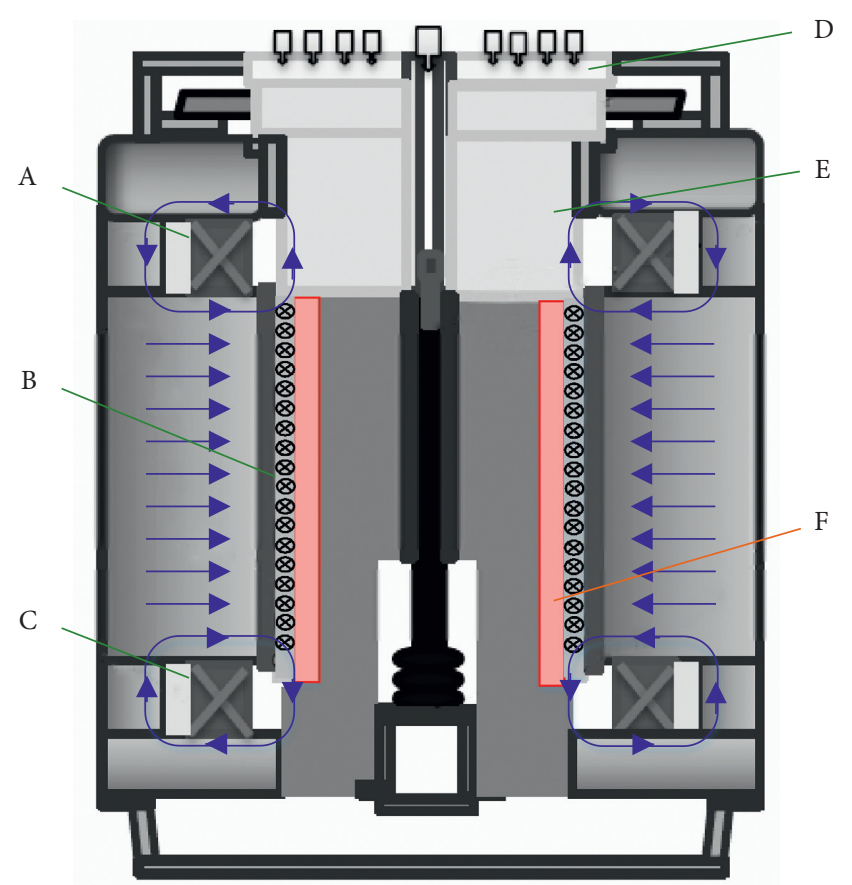

A: Upper excitation coil

B: Winding coil

D: Armature platform

C: Lower excitation coil

E: Armature skeleton

F: Armature structure

Figure 1: Armature structure under electromagnetic environment.

stiffness distribution, we simplify the armature structure to the cylindrical shell structure with ring stiffeners, to facilitate reveal the dynamic characteristics by employing nonlinear shell theory, Hamilton principle, and perturbation analysis theory. The novelty of the study is to establish the dynamic model of stiffened cylindrical shells subjected to electromagnetic excitations, transverse excitations, and in-plane excitations for the first time, and the current studies can provide a heuristic way for space engineers to realize the nonlinear vibrations of armature structure in a macroscopic view.

Different from the regular cylindrical shells, the structural traits of stiffened cylindrical shell are closer to the real engineering structure (i.e., armature structure), and thereby the stiffened cylindrical shell can perform more accurately the nonlinear vibration analysis from geometric modeling perspective. Up till now, stiffened cylindrical shells have been widely used in the civil, mechanical, aerospace, and aeronautic engineering fields [9-13]. The nonlinear shell theories have been studied in the past several decades [14]. Just like Donnell's shell theory $[15,16]$ and Sanders' shell theory $[17,18]$, Novozhilov theory $[19,20]$, Koiter theory $[21,22]$, and Flügge-Lur'e-Byrne theory [23, 24], which are classic shell theories, assure the computing efficiency in dynamic equation establishment for thin cylindrical shells. To describe the nonlinear vibrations of thin shells accurately, the first-order shear deformation theory [25-27] and the third-order shear deformation theory [28-30] are usually applied. Amabili [31] analyzed the nonlinear vibration of cylindrical shells with simply supported boundary and retained all the nonlinear terms of displacement and rotation in plane by using first-order shear deformation theory. Yazdi [32] studied the large amplitude vibration of composite hyperbolic shells considering von Karman's geometric nonlinearity and first-order shear deformation theory. Karimiasl [33] studied large amplitude vibration behaviors of multiscale doubly curved shells with piezoelectric layer by adopting Reddy's third-order shear deformation theory. Gu et al. [34] explored the nonlinear dynamics of circular cylindrical shells considering small initial geometric imperfection. Amabili and Reddy [35] improved the shell deformation theory to consider the rotation inertia terms, the shear deformation terms, and the nonlinear terms of the in-plane and lateral nonlinear displacements. According to these investigations, it is found that different nonlinear shell theories have different application scenarios. Moreover, due to the existence of stiffeners, the nonlinear behavior will produce some different characteristics and result in serious consequences, such as the bifurcation point drifts backward, and if the current theories and analysis results of regular cylindrical shells are directly used for stiffened cylindrical shells, unexpected deviations will arise. Therefore, it is significant for us to choose the appropriate nonlinear shell theory according to the geometric configuration of stiffened cylindrical shells.

Under the coupling effects of various complex excitations, the nonlinear vibrations and bifurcation phenomenon of stiffened cylindrical shells would be incurred and may lead to significant noises and resonance issues [36-38]. At present, there are more and more researches focusing on the frequency, chaotic motions, and instability for circular cylindrical shells under complex multiple excitations, and fruitful results have been achieved. Goncalves et al. [39] investigated the nonlinear vibration of cylindrical shells subjected to pulsating axial loads by using Donnell's shadow shell equation. Liu et al. [40] studied the bifurcation, intermittent chaos, and nonlinear vibration of a large deployable space antenna under $1: 3$ internal resonant thermal load. Dogan [41] established nonlinear response model for double-wall Sandwich cylindrical shells under random excitation and studied the parameter sensitivity on the nonlinear responses. Kumar et al. [42] studied the nonlinear response of elliptical shell under transverse harmonic load. Duc et al. [43] investigated the nonlinear response of functionally graded cylindrical shell with a ceramic metalceramic layer under uniformly distributed radial loads. Breslavsky et al. [44] studied that the dynamic behavior of cylindrical shells subjected to multiharmonic excitation is particularly complex and exhibits many types of nonlinear behaviors: simple periodic vibration, quasiperiodic oscillations, subharmonic response, period-doubling bifurcations, and chaos.

According to these researches on circular cylindrical shells, we find that the main objective of the current researches aims at investigating the regular cylindrical shells subjected to radial or/and in-plain excitations. However, multiscale composites, doubly curved shells and circular cylindrical shells with longitudinal or circumferential stiffeners, are not conventional in engineering practice. The 
existence of stiffeners will affect the dynamic characteristics of stiffened cylindrical shells and significantly increase the analysis complexity compared to that of ordinary cylindrical shells without stiffeners $[45,46]$. Moreover, stiffened cylindrical shells operating in electromagnetic environment usually suffer the coupling effects of transverse excitations, in-plain excitations, and electromagnetic excitations. Without considering complex multiple excitations in one unified framework, it will bring about unacceptable analysis deviations when describing the complex amplitude-frequency characteristics and chaotic motion behaviors [47-49]. Especially subjected to the strong electromagnetic environment, it is evitable to bring in unacceptable analysis deviations if the electromagnetic excitation and various external excitations are not considered simultaneously.

In this paper, the nonlinear vibrations of stiffened cylindrical shells subjected to electromagnetic environment are studied from a theoretical analysis view. A dynamic model of stiffened cylindrical shells subjected to transverse excitations, in-plane excitations, and electromagnetic excitations is proposed for the first time. To begin with, in the framework of the first-order shear deformation shell theory, the governing equations of motion for stiffened cylindrical shells are derived by using Hamilton's principle. Then, the governing equations of motion are discretized into nonlinear ordinary differential governing equations by utilizing the Galerkin discretization procedure. The multiscale method of perturbation analysis is employed to obtain fourdimensional nonlinear averaged equations under the $1: 2$ internal resonance and principal resonance-1/2 subharmonic parametric resonance. Moreover, the nonlinear dynamic behaviors and the jump phenomena are revealed in the amplitude-frequency curves by considering the effects of geometric parameters and multiple excitations. Lastly, the periodic and chaotic motions of the stiffened cylindrical shell are investigated by Runge-Kutta approach, and the influence of stiffener number, stiffener size, and aspect ratio on the nonlinear dynamic responses of the stiffened cylindrical shell is acquired.

\section{Dynamic Equations of Motion}

The simplified geometric model of stiffened cylindrical shell in an external uniform electromagnetic field $\left(0,0, B_{z}\right)$ is presented, as shown in Figure 2. The basic parameters of stiffened cylindrical shell involve shell length $L$, shell middle surface radius $R$, shell thickness $h$, stiffener height $h_{s}$, stiffener thickness $d_{s}$, and stiffener spacing $b_{s}$. To analyze the vibration characteristics of the stiffened cylindrical shell, the cylindrical coordinate system $(x, \theta, z)$ is built on the left-edge middle surface. Herein, $x$ denotes the axis direction of stiffened cylindrical shell, and $\theta$ and $z$ represent the circumferential and radial directions of the stiffened cylindrical shell.

To describe the vibration behaviors of stiffened cylindrical shells, by considering the transverse shear deformation, Reddy's first-order shear deformation theory-based [25-27] dynamic model is constructed. Assuming that the transverse normal would not remain normal to the middle surface after shell deformation, the displacement field is established:

$$
\left\{\begin{array}{l}
u(x, \theta, z, t)=u_{0}(x, \theta, t)+z \phi_{x}(x, \theta, t), \\
v(x, \theta, z, t)=v_{0}(x, \theta, t)+z \phi_{\theta}(x, \theta, t), \\
w(x, \theta, z, t)=w_{0}(x, \theta, t)
\end{array}\right.
$$

where $u_{0}, v_{0}$, and $w_{0}$ indicate middle surface displacements along the $x$-axis, $\theta$-axis, and $z$-axis, respectively; $\phi_{x}$ and $\phi_{\theta}$ are the rotation angle along the $\theta$-axis and $x$-axis, respectively; $t$ is the time variable. With considering the von Karman geometric nonlinearity rule [50], the geometric equation is built:

$$
\left\{\begin{array}{c}
\varepsilon_{x x} \\
\varepsilon_{\theta \theta} \\
\gamma_{x \theta} \\
\gamma_{x z} \\
\gamma_{\theta z}
\end{array}\right\}=\left\{\begin{array}{c}
\varepsilon_{x x}^{0} \\
\varepsilon_{\theta \theta}^{0} \\
\gamma_{x \theta}^{0} \\
\gamma_{x z}^{0} \\
\gamma_{\theta z}^{0}
\end{array}\right\}+z\left\{\begin{array}{c}
\varepsilon_{x x}^{1} \\
\varepsilon_{\theta \theta}^{1} \\
\gamma_{x \theta}^{1} \\
\gamma_{x z}^{1} \\
\gamma_{\theta z}^{1}
\end{array}\right\},
$$

$$
\left\{\begin{array}{c}
\varepsilon_{x x}^{0} \\
\varepsilon_{\theta \theta}^{0} \\
\gamma_{x \theta}^{0} \\
\gamma_{x z}^{0} \\
\gamma_{\theta z}^{0}
\end{array}\right\}=\left\{\begin{array}{c}
\frac{\partial u_{0}}{\partial x}+\frac{1}{2}\left(\frac{\partial w_{0}}{\partial x}\right)^{2} \\
\frac{\partial v_{0}}{R \partial \theta}+\frac{1}{2}\left(\frac{\partial w_{0}}{R \partial \theta}\right)^{2}+\frac{w_{0}}{R}+\frac{\partial v_{0}}{\partial x}+\frac{\partial w_{0}}{\partial x} \frac{\partial w_{0}}{R \partial \theta} \\
\phi_{x}+\frac{\partial w_{0}}{\partial x} \\
\phi_{\theta}+\frac{\partial w_{0}}{R \partial \theta}
\end{array}\right\},
$$

$$
\left\{\begin{array}{c}
\varepsilon_{x x}^{1} \\
\varepsilon_{\theta \theta}^{1} \\
\gamma_{x \theta}^{1} \\
\gamma_{x z}^{1} \\
\gamma_{\theta z}^{1}
\end{array}\right\}=\left\{\begin{array}{c}
\frac{\partial \phi_{x}}{\partial x} \\
\frac{\partial \phi_{\theta}}{R \partial \theta} \\
\frac{\partial \phi_{x}}{R \partial \theta}+\frac{\partial \phi_{\theta}}{\partial x} \\
0 \\
0
\end{array}\right\}
$$

where $\varepsilon_{x x}, \varepsilon_{\theta \theta}, \gamma_{x \theta}, \gamma_{x z}$, and $\gamma_{\theta z}$ denote the strain components; $\left(\varepsilon_{x x}^{0}, \varepsilon_{\theta \theta}^{0}, \gamma_{x \theta}^{0}, \gamma_{x z}^{0}, \gamma_{\theta z}^{0}\right)$ are the membrane strains; $\left(\varepsilon_{x x}^{1}, \varepsilon_{\theta \theta}^{1}\right.$, $\left.\gamma_{x \theta}^{1}, \gamma_{x z}^{1}, \gamma_{\theta z}^{1}\right)$ are the flexural strains. 

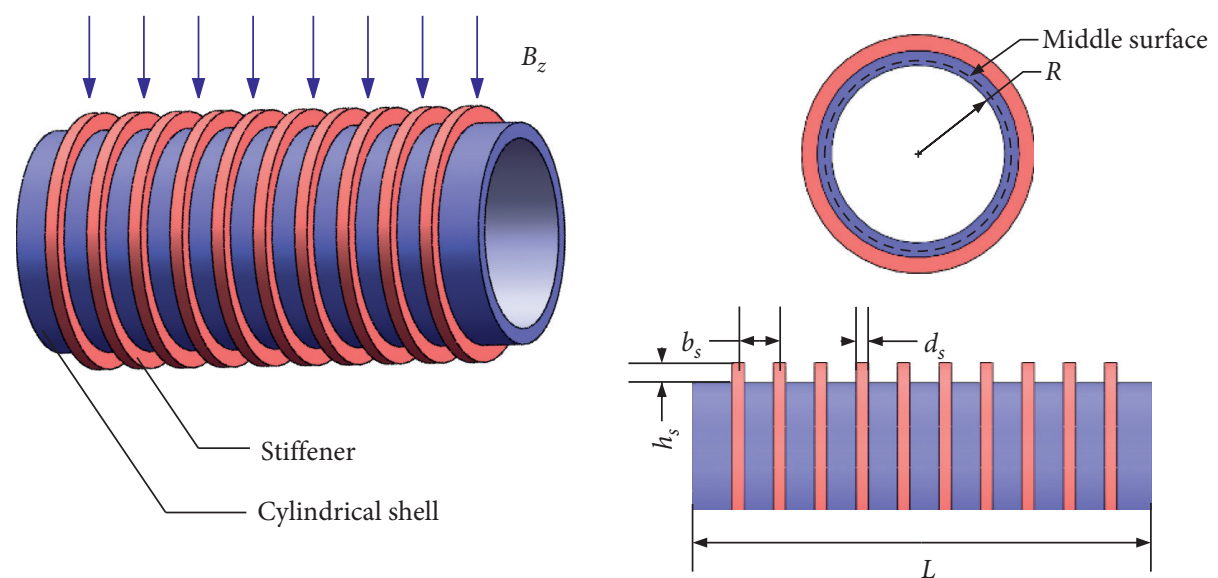

Figure 2: Simplified geometric model of stiffened cylindrical shell.

In light of the general Hook's law, to further reveal the stress behavior in terms of displacement, the constitutive relation of stiffened cylindrical shells is indicated:

$$
\left\{\begin{array}{c}
\sigma_{x x} \\
\sigma_{\theta \theta} \\
\tau_{x \theta} \\
\tau_{x z} \\
\tau_{\theta z}
\end{array}\right\}=\left\{\begin{array}{lllll}
Q_{11} & Q_{12} & & & \\
Q_{12} & Q_{22} & & & \\
& & Q_{44} & \\
& & & Q_{55} & \\
& & & & Q_{66}
\end{array}\right\}\left\{\begin{array}{c}
\varepsilon_{x x} \\
\varepsilon_{\theta \theta} \\
\gamma_{x \theta} \\
\gamma_{x z} \\
\gamma_{\theta z}
\end{array}\right\},
$$

where $Q_{i j}(i=1,2$ and $j=1,2 ; i=4-6$ and $j=4-6)$ are the elastic constants of stiffened cylindrical shell:

$$
\begin{aligned}
& Q_{11}=Q_{22}=\frac{E}{1-v^{2}}, \\
& Q_{12}=v \frac{E}{1-v^{2}}, \\
& Q_{44}=Q_{55}=Q_{66}=\frac{E}{2(1+v)}, \\
& \sigma_{\theta \theta}^{s}=E^{s} \varepsilon_{\theta \theta},
\end{aligned}
$$

in which $\left(\sigma_{x x}, \sigma_{\theta \theta}, \sigma_{\theta \theta}^{s}\right)$ are the coefficients of the normal stress; $\tau_{x \theta}$ are the coefficients of the shear stress; $\left(\tau_{x z}, \tau_{\theta z}\right)$ are the coefficients of the bending stress; $E$ is Young's modulus of the cylindrical shell; $E^{\mathcal{S}}$ is Young's modulus of stiffeners.

The force and bending moment distributions on middle surface of cylindrical shells are shown in Figure 3, and they can be quantified in equation (5).

$$
\begin{aligned}
N_{x x} & =\int_{-(h / 2)}^{h / 2} \sigma_{x x} \mathrm{~d} z, \\
M_{x x} & =\int_{-(h / 2)}^{h / 2} \sigma_{x x} z \mathrm{~d} z, \\
N_{\theta \theta} & =\int_{-(h / 2)}^{h / 2} \sigma_{\theta \theta} \mathrm{d} z, \\
N_{\theta \theta}^{s} & +\int_{h / 2}^{(h / 2)+h_{s}} \sigma_{\theta \theta} \mathrm{d} z, \\
N_{x \theta} & =\int_{-(h / 2)}^{h / 2} \tau_{x \theta} \mathrm{d} z, \\
M_{\theta \theta} & =\int_{-(h / 2)}^{h / 2} \sigma_{\theta \theta} z \mathrm{~d} z, \\
M_{\theta \theta}^{s} & =\int_{h / 2}^{(h / 2)+h_{s}} \sigma_{\theta \theta} z \mathrm{~d} z, \\
M_{x \theta} & =\int_{-(h / 2)}^{h / 2} \tau_{x \theta} z \mathrm{~d} z, \\
Q_{x} & =\int_{-(h / 2)}^{h / 2} \tau_{x z} \mathrm{~d} z, \\
Q_{\theta} & =\int_{-(h / 2)}^{h / 2} \tau_{\theta z} \mathrm{~d} z .
\end{aligned}
$$

As operating in electromagnetic environment, stiffened cylindrical shells make deformation under the electromagnetic force, and the structural deformation further leads to the change of distribution condition of electromagnetic flux and electromagnetic force. These coupling effects between the electromagnetic field and the mechanical field bring in the high nonlinearity and large time-varying characteristics, which leads to great difficulty in performing the quantitative analysis of mechanical behavior for stiffened cylindrical shells. In this study, to clearly describe the mechanical 


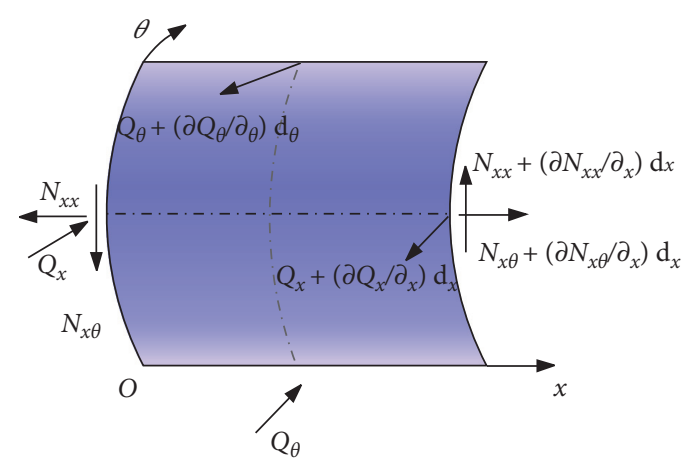

(a)

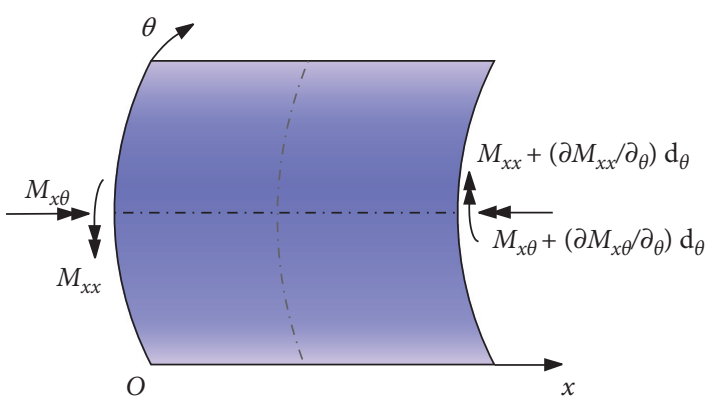

(b)

Figure 3: (a) Force distribution and (b) bending moment distribution on middle surface of the cylindrical shell.

behavior of stiffened cylindrical shells, considering the influence of electromagnetic force on virtual work under external excitations, the dynamic equations of motion under electromagnetic environment are established. By introducing the Lorentz law of force [51], the electromagnetic force acting on stiffened cylindrical shells is presented as follows.

Based on the law of electromagnetic induction [52], the electric current induced by cylindrical shell motion in radial electromagnetic field can be expressed as

$$
\mathbf{J}=\sigma(\mathbf{V} \times \mathbf{B})=\sigma\left(\frac{\partial \mathbf{u}}{\partial t} \times \mathbf{B}\right)
$$

where $\mathbf{J}$ denotes the electric current vectors, $\sigma$ denotes the conductivity, $\mathbf{V}$ denotes the speed, and $\mathbf{B}$ denotes the electromagnetic intensity, where $\mathbf{B}=\left(0,0, B_{z}\right)$. Therein, $i, j$, and $k$ are regarded as the vector in the three directions of $x, \theta$, and $z$; then, the displacement vector of the stiffened cylindrical shell $\mathbf{u}$ can be expressed as

$$
\mathbf{u}=\left(u_{0}+z \phi_{x}\right) i+\left(v_{0}+z \phi_{\theta}\right) j+w_{0}(x, \theta, t) k
$$

In the constant electromagnetic field, the Lorentz electromagnetic force per unit volume can be expressed as

$$
\begin{aligned}
\mathbf{f}\left(f_{u}, f_{v}, f_{w}\right) & =\mathbf{J} \times \mathbf{B}=\sigma\left(\frac{\partial u}{\partial t} \times \mathbf{B}\right) \times \mathbf{B} \\
& =\sigma\left[\left(\left(\frac{\partial u_{0}}{\partial t}+z \frac{\partial \phi_{x}}{\partial t}\right) i,\left(\frac{\partial v_{0}}{\partial t}+z \frac{\partial \phi_{\theta}}{\partial t}\right) j, \frac{\partial w_{0}}{\partial t} k\right) \times\left(0,0, B_{z} k\right)\right] \times\left(0,0, B_{z} k\right) \\
& =\left(\left(-\sigma B_{z}^{2}\left(\frac{\partial u_{0}}{\partial t}+z \frac{\partial \phi_{x}}{\partial t}\right)\right) i,\left(-\sigma B_{z}^{2}\left(\frac{\partial v_{0}}{\partial t}+z \frac{\partial \phi_{\theta}}{\partial t}\right)\right) j, 0\right) \\
& =f_{u} i+f_{v} j .
\end{aligned}
$$

Furtherly, the Lorentz forces $F_{x}$ and $F_{\theta}$ (in $x$ and $\theta$-axis) and electromagnetic moments $M_{x}$ and $M_{\theta}$ (in $\theta$ and $x$-axis) can be derived as follows: 


$$
\begin{aligned}
& \left\{\begin{array}{l}
F_{x}=\int_{-(h / 2)}^{h / 2} f_{u} \mathrm{~d} z \\
=-\sigma B_{z}^{2} \frac{\partial u_{0}}{\partial t} h, \\
F_{\theta}=\int_{-(h / 2)}^{h / 2} f_{v} \mathrm{~d} z \\
=-\sigma B_{z}^{2} \frac{\partial v_{0}}{\partial t} h, \\
M_{x}=\int_{-(h / 2)}^{h / 2} f_{u} z \mathrm{~d} z \\
=\frac{\sigma h^{3} B_{z}^{2}}{12} \frac{\partial \phi_{x}}{\partial t}, \\
M_{\theta}=\int_{-(h / 2)}^{h / 2} f_{v} z \mathrm{~d} z \\
=\frac{\sigma h^{3} B_{z}^{2}}{12} \frac{\partial \phi_{\theta}}{\partial t} .
\end{array}\right.
\end{aligned}
$$

Based on the nonlinear constitutive equation and the magnetic force described by the Lorentz law of force, the nonlinear dynamic equation of the system can be established. Given Hamilton's principle [53], the nonlinear dynamic equation of stiffened cylindrical shell under electromagnetic environment is established as follows:

$$
\int_{t_{2}}^{t_{1}}[\delta(T-U)+\delta W] \mathrm{d} t=0 .
$$

Therein, the kinetic energy $\delta T$, potential energy $\delta U$, and virtual work $\delta W$ under external excitations can be signified as follows:

$$
\begin{aligned}
& \delta T=\int_{V} \rho(\dot{u} \delta \dot{u}, \dot{v} \delta \dot{v}, \dot{w} \delta \dot{w}) \mathrm{d} V+\int_{V s} \frac{d_{s}}{b_{s}} \rho(\dot{u} \delta \dot{u}, \dot{v} \delta \dot{v}, \dot{w} \delta \dot{w}) \mathrm{d} V s, \\
& \delta U=\int_{V}\left(\sigma_{x x} \delta \varepsilon_{x x}+\sigma_{\theta \theta} \delta \varepsilon_{\theta \theta}+\sigma_{x \theta} \delta \varepsilon_{x \theta}+\sigma_{x z} \delta \varepsilon_{x z}+\sigma_{\theta z} \delta \varepsilon_{\theta z}\right) \mathrm{d} V+\int_{V_{s}} \frac{d_{s}}{b_{s}} \sigma_{\theta \theta} \delta \varepsilon_{\theta \theta} \mathrm{d} V s, \\
& \delta W=\int_{V}\left(F_{x} \delta u_{0}+F_{\theta} \delta v_{0}+M_{x} \delta \phi_{x}+M_{\theta} \delta \phi_{\theta}\right) \mathrm{d} V+\int_{A} N w_{0, x x} \delta w_{0} \mathrm{~d} A+\int_{A} F \delta w_{0} \mathrm{~d} A,
\end{aligned}
$$

where $N=N_{1} \sin \left(\omega_{1} t\right)$ represents the in-plane excitation, $N_{1}$ represents the in-plane excitation amplitude, $F=F_{1} \sin \left(\omega_{2} t\right)$ represents the time-depend transverse excitation, $F_{1}$ represents the transverse excitation amplitude, $\omega_{1}$ represents the in-plane excitation frequency, and $\omega_{2}$ represents the transverse excitation frequency.

The nonlinear partial differential governing equations of motion for stiffened cylindrical shell are given by 
substituting the kinetic energy, potential energy, and virtual work into Hamilton's equation:

$$
\left\{\begin{array}{l}
\delta u_{0}: N_{x x, x}+\frac{N_{x \theta, \theta}}{R}=I_{0} \ddot{u}_{0}+I_{1} \ddot{\phi}_{x}+\sigma B_{z}^{2} \dot{u}_{0} h, \\
\delta v_{0}: \frac{N_{\theta \theta, \theta}}{R}+N_{x \theta, x}+N_{\theta \theta, \theta}^{s}=I_{1} \ddot{\phi}_{\theta}+I_{0} \ddot{v}_{0}+\sigma B_{z}^{2} \dot{v}_{0} h, \\
\delta w_{0}: N_{x x, x} \frac{\partial w_{0}}{\partial x}+N_{\theta \theta, \theta}^{s} \frac{\partial w_{0}}{\partial x}+N_{x x} \frac{\partial^{2} w_{0}}{\partial x^{2}}+N_{\theta \theta}^{s} \frac{\partial^{2} w_{0}}{\partial x^{2}}+\frac{N_{\theta \theta, \theta}}{R} \frac{\partial w_{0}}{R \partial \theta} \\
+N_{\theta \theta} \frac{\partial^{2} w_{0}}{R^{2} \partial \theta^{2}}-\frac{N_{\theta \theta}}{R}+N_{x \theta, x} \frac{\partial w_{0}}{R \partial \theta}+N_{x \theta} \frac{\partial^{2} w_{0}}{R \partial \theta}+\frac{N_{x \theta \theta \theta}}{R} \frac{\partial w_{0}}{\partial x} \\
+N_{x \theta} \frac{\partial^{2} w_{0}}{R \partial \theta}+Q_{x, x}+\frac{Q_{\theta, \theta}}{R}-N \frac{\partial^{2} w_{0}}{\partial x^{2}}+F=I_{0} \ddot{w}_{0}, \\
\delta \phi_{x}: \frac{M_{x \theta, \theta}}{R}+M_{x x, x}-Q_{x}=I_{2} \ddot{\phi}_{x}+I_{1} \ddot{u}_{0}-\frac{\sigma h^{3} B_{z}^{2}}{12} \dot{\phi}_{x}, \\
\delta \phi_{\theta}: \frac{M_{\theta \theta, \theta}}{R}+M_{x \theta, x}+M_{\theta \theta, \theta}^{s}-Q_{\theta}=I_{1} \ddot{v}_{0}+I_{2} \ddot{\phi}_{\theta}-\frac{\sigma h^{3} B_{z}^{2}}{12} \dot{\phi}_{\theta} .
\end{array}\right.
$$

By substituting the force and moment resultants (5) into equation (13), the governing equations of motion in the generalized displacements $\left(u_{0}, v_{0}\right.$, and $\left.w_{0}\right)$ can be rewritten as

$$
\begin{gathered}
A_{11} \frac{\partial^{2} u_{0}}{\partial x^{2}}+A_{11} \frac{\partial w_{0}}{\partial x} \frac{\partial^{2} w_{0}}{\partial x^{2}}+A_{12} \frac{\partial^{2} v_{0}}{R \partial \theta \partial x}+A_{12} \frac{\partial w_{0}}{R \partial \theta} \frac{\partial^{2} w_{0}}{R \partial \theta}+A_{12} \frac{\partial w_{0}}{R x}+B_{11} \frac{\partial^{2} \phi_{x}}{\partial x^{2}}+B_{s} \frac{\partial^{2} \phi_{x}}{\partial x^{2}}+A_{s} \frac{\partial^{2} u_{0}}{\partial x^{2}}+A_{s} \frac{\partial w_{0}}{\partial x} \frac{\partial^{2} w_{0}}{\partial x^{2}} \\
+B_{12} \frac{\partial^{2} \phi_{\theta}}{R \partial \theta \partial x}+A_{33} \frac{\partial^{2} u_{0}}{R^{2} \partial \theta^{2}}+A_{33} \frac{\partial^{2} v_{0}}{R \partial \theta}+A_{33} \frac{\partial^{2} w_{0}}{R \partial \theta \partial x} \frac{\partial w_{0}}{R \partial \theta}+A_{33} \frac{\partial w_{0}}{\partial x} \frac{\partial^{2} w_{0}}{R^{2} \partial \theta^{2}}+B_{33} \frac{\partial^{2} \phi_{x}}{R^{2} \partial \theta^{2}}+B_{33} \frac{\partial^{2} \phi_{\theta}}{R \partial \theta}=I_{0} \ddot{u}_{0}+I_{1} \ddot{\phi}_{x}, \\
A_{12} \frac{\partial^{2} u_{0}}{R \partial x \partial \theta}+A_{12} \frac{\partial w_{0}}{\partial x} \frac{\partial^{2} w_{0}}{R \partial \theta \partial x}+A_{22} \frac{\partial^{2} v_{0}}{R^{2} \partial \theta^{2}}+A_{22} \frac{\partial w_{0}}{R \partial \theta} \frac{\partial^{2} w_{0}}{R^{2} \partial \theta^{2}}+A_{22} \frac{\partial w_{0}}{R^{2} \partial \theta}+B_{12} \frac{\partial^{2} \phi_{x}}{R \partial \theta \partial x}+B_{22} \frac{\partial^{2} \phi_{\theta}}{R^{2} \partial \theta^{2}} \\
+A_{33} \frac{\partial^{2} u_{0}}{R \partial \theta \partial x}+A_{33} \frac{\partial^{2} v_{0}}{\partial x^{2}}+A_{33} \frac{\partial^{2} w_{0}}{\partial x^{2}} \frac{\partial w_{0}}{R \partial \theta}+A_{33} \frac{\partial w_{0}}{\partial x} \frac{\partial^{2} w_{0}}{R \partial \theta \partial x}+B_{33} \frac{\partial^{2} \phi_{x}}{R \partial \theta \partial x}+B_{33} \frac{\partial^{2} \phi_{\theta}}{\partial x^{2}}=I_{1} \ddot{\phi}_{\theta}+I_{0} v_{0},
\end{gathered}
$$




$$
\begin{aligned}
& A_{s} \frac{\partial^{2} u_{0}}{\partial x^{2}} \frac{\partial w_{0}}{\partial x}+A_{s} \frac{\partial w_{0}}{\partial x} \frac{\partial^{2} w_{0}}{\partial x^{2}} \frac{\partial w_{0}}{\partial x}+B_{s} \frac{\partial^{2} \phi_{x}}{\partial x^{2}} \frac{\partial w_{0}}{\partial x}+A_{s} \frac{\partial u_{0}}{\partial x} \frac{\partial^{2} w_{0}}{\partial x^{2}}+A_{s} \frac{1}{2}\left(\frac{\partial w_{0}}{\partial x}\right)^{2} \frac{\partial^{2} w_{0}}{\partial x^{2}}+B_{s} \frac{\partial \phi_{x}}{\partial x} \frac{\partial^{2} w_{0}}{\partial x^{2}} \frac{\partial w_{0}}{\partial x} \\
& +\left(A_{11} \frac{\partial^{2} u_{0}}{\partial x^{2}}+A_{11} \frac{\partial w_{0}}{\partial x} \frac{\partial^{2} w_{0}}{\partial x^{2}}+A_{12} \frac{\partial^{2} v_{0}}{R \partial \theta \partial x}+A_{12} \frac{\partial w_{0}}{R \partial \theta} \frac{\partial^{2} w_{0}}{R \partial \theta \partial x}+A_{12} \frac{\partial w_{0}}{R \partial x}+B_{11} \frac{\partial^{2} \phi_{x}}{\partial x^{2}}+B_{12} \frac{\partial^{2} \phi_{\theta}}{R \partial \theta \partial x}\right) \\
& +\left(A_{11} \frac{\partial u_{0}}{\partial x}+A_{11} \frac{1}{2}\left(\frac{\partial w_{0}}{\partial x}\right)^{2}+A_{12} \frac{\partial v_{0}}{R \partial \theta}+A_{12} \frac{1}{2}\left(\frac{\partial w_{0}}{R \partial \theta}\right)^{2}+A_{12} \frac{w_{0}}{R}+B_{11} \frac{\partial \phi_{x}}{\partial x}+B_{12} \frac{\partial \phi_{\theta}}{R \partial \theta}\right) \frac{\partial^{2} w_{0}}{\partial x^{2}} \\
& +\left(A_{12} \frac{\partial^{2} u_{0}}{R \partial x \partial \theta}+A_{12} \frac{\partial w_{0}}{\partial x} \frac{\partial^{2} w_{0}}{R \partial \theta \partial x}+A_{22} \frac{\partial^{2} v_{0}}{R^{2} \partial \theta^{2}}+A_{22} \frac{\partial w_{0}}{R \partial \theta} \frac{\partial^{2} w_{0}}{R^{2} \partial \theta^{2}}+A_{22} \frac{\partial w_{0}}{R^{2} \partial \theta}+B_{12} \frac{\partial^{2} \phi_{x}}{R \partial \theta \partial x}+B_{22} \frac{\partial^{2} \phi_{\theta}}{R^{2} \partial \theta^{2}}\right) \frac{\partial w_{0}}{R \partial \theta} \\
& +\left(A_{12} \frac{\partial u_{0}}{\partial x}+A_{12} \frac{1}{2}\left(\frac{\partial w_{0}}{\partial x}\right)^{2}+A_{22} \frac{\partial v_{0}}{R \partial \theta}+A_{22} \frac{1}{2}\left(\frac{\partial w_{0}}{R \partial \theta}\right)^{2}+A_{22} \frac{w_{0}}{R}+B_{12} \frac{\partial \phi_{x}}{\partial x}+B_{22} \frac{\partial \phi_{\theta}}{R \partial \theta}\right) \frac{\partial^{2} w_{0}}{R^{2} \partial \theta^{2}} \\
& -\frac{1}{R}\left(A_{12} \frac{\partial u_{0}}{\partial x}+A_{12} \frac{1}{2}\left(\frac{\partial w_{0}}{\partial x}\right)^{2}+A_{22} \frac{\partial v_{0}}{R \partial \theta}+A_{22} \frac{1}{2}\left(\frac{\partial w_{0}}{R \partial \theta}\right)^{2}+A_{22} \frac{w_{0}}{R}+B_{12} \frac{\partial \phi_{x}}{\partial x}+B_{22} \frac{\partial \phi_{\theta}}{R \partial \theta}\right) \\
& +\left(A_{33} \frac{\partial^{2} u_{0}}{R \partial \theta \partial x}+A_{33} \frac{\partial^{2} v_{0}}{\partial x^{2}}+A_{33} \frac{\partial^{2} w_{0}}{\partial x^{2}} \frac{\partial w_{0}}{R \partial \theta}+A_{33} \frac{\partial w_{0}}{\partial x} \frac{\partial^{2} w_{0}}{R \partial \theta \partial x}+B_{33} \frac{\partial^{2} \phi_{x}}{R \partial \theta \partial x}+B_{33} \frac{\partial^{2} \phi_{\theta}}{\partial x^{2}}\right) \frac{\partial w_{0}}{R \partial \theta} \\
& +\left(A_{33} \frac{\partial u_{0}}{R \partial \theta}+A_{33} \frac{\partial v_{0}}{\partial x}+A_{33} \frac{\partial w_{0}}{\partial x} \frac{\partial w_{0}}{R \partial \theta}+B_{33} \frac{\partial \phi_{x}}{R \partial \theta}+B_{33} \frac{\partial \phi_{\theta}}{\partial x}\right) \frac{\partial^{2} w_{0}}{R \partial \theta \partial x} \\
& +\left(A_{33} \frac{\partial^{2} u_{0}}{R^{2} \partial \theta^{2}}+A_{33} \frac{\partial^{2} v_{0}}{R \partial x \partial \theta}+A_{33} \frac{\partial^{2} w_{0}}{\partial x R \partial \theta} \frac{\partial w_{0}}{R \partial \theta}+A_{33} \frac{\partial w_{0}}{\partial x} \frac{\partial^{2} w_{0}}{R^{2} \partial \theta^{2}}+B_{33} \frac{\partial^{2} \phi_{x}}{R^{2} \partial \theta^{2}}+B_{33} \frac{\partial^{2} \phi_{\theta}}{R \partial x \partial \theta}\right) \frac{\partial w_{0}}{\partial x} \\
& +\left(A_{33} \frac{\partial u_{0}}{R \partial \theta}+A_{33} \frac{\partial v_{0}}{\partial x}+A_{33} \frac{\partial w_{0}}{\partial x} \frac{\partial w_{0}}{R \partial \theta}+B_{33} \frac{\partial \phi_{x}}{R \partial \theta}+B_{33} \frac{\partial \phi_{\theta}}{\partial x}\right) \frac{\partial^{2} w_{0}}{R \partial \theta \partial x} \\
& +A_{44} \frac{\partial \phi_{x}}{\partial x}+A_{44} \frac{\partial^{2} w_{0}}{\partial x^{2}}+A_{55} \frac{\partial \phi_{\theta}}{R \partial \theta}+A_{55} \frac{\partial^{2} w_{0}}{R^{2} \partial \theta^{2}}+\frac{B_{z}^{2} h}{2 \mu_{0}}\left[\left(\frac{\partial w_{0}}{\partial x}\right)^{2}+\left(\frac{\partial w_{0}}{R \partial \theta}\right)^{2}\right] \\
& +\rho g h \frac{\partial w_{0}}{\partial x}-\rho g h(L-x) \frac{\partial^{2} w_{0}}{\partial x^{2}}-N \frac{\partial^{2} w_{0}}{\partial x^{2}}+F=I_{0} \ddot{w}_{0}
\end{aligned}
$$$$
B_{s} \frac{\partial^{2} u_{0}}{\partial x^{2}}+B_{s} \frac{\partial w_{0}}{\partial x} \frac{\partial^{2} w_{0}}{\partial x^{2}}+D_{s} \frac{\partial^{2} \phi_{x}}{\partial x^{2}}+B_{33} \frac{\partial^{2} u_{0}}{R^{2} \partial \theta^{2}}+B_{33} \frac{\partial^{2} v_{0}}{R \partial \theta \partial x}+B_{33} \frac{\partial^{2} w_{0}}{R \partial \theta \partial x} \frac{\partial w_{0}}{R \partial \theta}+B_{33} \frac{\partial w_{0}}{\partial x} \frac{\partial^{2} w_{0}}{R^{2} \partial \theta^{2}}
$$$$
+D_{33} \frac{\partial^{2} \phi_{x}}{R^{2} \partial \theta^{2}}+D_{33} \frac{\partial^{2} \phi_{\theta}}{R \partial \theta \partial x}+B_{11} \frac{\partial^{2} u_{0}}{\partial x^{2}}+B_{11} \frac{\partial w_{0}}{\partial x} \frac{\partial^{2} w_{0}}{\partial x^{2}}+B_{12} \frac{\partial^{2} v_{0}}{R \partial \theta \partial x}+B_{12} \frac{\partial w_{0}}{R \partial \theta} \frac{\partial^{2} w_{0}}{R \partial \theta \partial x}+B_{12} \frac{\partial w_{0}}{R \partial x},
$$$$
+D_{11} \frac{\partial^{2} \phi_{x}}{\partial x^{2}}+D_{12} \frac{\partial^{2} \phi_{\theta}}{R \partial \theta \partial x}-A_{44} \phi_{x}-A_{44} \frac{\partial w_{0}}{\partial x}+\frac{\sigma h^{3} B_{z}^{2}}{12}\left(\frac{\partial u_{0}}{\partial t}+z \frac{\partial \phi_{x}}{\partial t}\right)=I_{2} \ddot{\phi}_{x}-I_{1} \ddot{u}_{0}
$$

$$
B_{12} \frac{\partial^{2} u_{0}}{R \partial x \partial \theta}+B_{12} \frac{\partial w_{0}}{\partial x} \frac{\partial^{2} w_{0}}{R \partial \theta \partial x}+B_{22} \frac{\partial^{2} v_{0}}{R^{2} \partial \theta^{2}}+B_{22} \frac{\partial w_{0}}{R \partial \theta} \frac{\partial^{2} w_{0}}{R^{2} \partial \theta^{2}}+B_{22} \frac{\partial w_{0}}{R^{2} \partial \theta}+D_{12} \frac{\partial^{2} \phi_{x}}{R \partial x \partial \theta}
$$

$$
\begin{aligned}
& +D_{22} \frac{\partial^{2} \phi_{\theta}}{R^{2} \partial \theta^{2}}+B_{33} \frac{\partial^{2} u_{0}}{R \partial \theta \partial x}+B_{33} \frac{\partial^{2} v_{0}}{\partial x^{2}}+B_{33} \frac{\partial^{2} w_{0}}{\partial x^{2}} \frac{\partial w_{0}}{R \partial \theta}+B_{33} \frac{\partial w_{0}}{\partial x} \frac{\partial^{2} w_{0}}{R \partial \theta \partial x}+D_{33} \frac{\partial^{2} \phi_{x}}{R \partial \theta \partial x}+D_{33} \frac{\partial^{2} \phi_{\theta}}{\partial x^{2}}+I_{2} \ddot{\phi}_{\theta} \\
& -A_{55} \phi_{\theta}-A_{55} \frac{\partial w_{0}}{R \partial \theta}+\frac{\sigma h^{3} B_{z}^{2}}{12}\left(\frac{\partial v_{0}}{\partial t}+z \frac{\partial \phi_{\theta}}{\partial t}\right)=-I_{1} \ddot{v}_{0}
\end{aligned}
$$

Note that the parts which involve magnetic field strength $B_{Z}$ are used to describe the contribution of the electromagnetic environment. Moreover, the coefficients $A_{i j}$, $B_{i j}, D_{i j}, A_{s}, B_{s}$, and $D_{s}$ can be further expressed as follows: 


$$
\begin{aligned}
\left(A_{i j}, B_{i j}, D_{i j}\right) & =\int_{-(h / 2)}^{h / 2} Q_{i j}\left(1, z, z^{2}\right) \mathrm{d} z, \\
\left(A_{s}, B_{s}, D_{s}\right) & =\int_{h / 2}^{(h / 2)+h_{s}} E^{s} \frac{b_{s}}{d_{s}}\left(1, z, z^{2}\right) \mathrm{d} z .
\end{aligned}
$$

\section{Equations' Discretization and Perturbation Analysis}

In this section, a two-step solution procedure is considered to solve the nonlinear dynamic equations of the stiffened cylindrical shell: (i) the Galerkin discretization approach is applied to divert the partial differential equations into ordinary differential equations; (ii) the multiscale method of perturbation analysis is adopted to obtain the four-dimensional average equation of the system. The solution procedures of the nonlinear dynamic equations are summarized as follows.

Considering the deformation features of armature structures, simply supported boundaries are employed to constrain both ends of the stiffened cylindrical shell, which can be expressed as

$$
\begin{aligned}
v_{0} & =w_{0}=\phi_{\theta}=M_{x x}=0, \\
\left.\int_{0}^{2 \pi R} N_{x x}\right|_{x=0, L} \mathrm{~d} \theta & =\int_{0}^{2 \pi R} N \mathrm{~d} \theta, \quad \text { at } x=0, x=L,
\end{aligned}
$$

where $N_{0}$ represents the in-plane excitation.

Regarding the fact that in-plane inertial terms are much smaller than the transverse inertial terms [54], the ordinary differential governing equations are derived by ignoring the in-plane inertial term in governing equations. Herein, the continuous system of the partial governing differential equation is truncated into a two-degree-of-freedom system of ordinary differential governing equations by using the Galerkin discretization approach, and the reasonable approximation functions are desired to expand the displacements in the middle surface. Since most of the vibration energy mainly concentrates in the first-order and secondorder modes, accounting for $90 \%$ or more of the system energy [55], the first two modes are taken into account to describe the nonlinear dynamic behaviors of stiffened cylindrical shells. Based on [56, 57], the displacements $u_{0}, v_{0}$, $w_{0}, \phi_{x}$, and $\phi_{\theta}$ which satisfy the boundary conditions are represented as

$$
\left\{\begin{array}{l}
u_{0}=U_{1}(t) \cos \left(\frac{\pi x}{L}\right) \cos (3 \theta)+U_{2}(t) \cos \left(\frac{3 \pi x}{L}\right) \cos (\theta), \\
v_{0}=V_{1}(t) \sin \left(\frac{\pi x}{L}\right) \cos (3 \theta)+V_{2}(t) \sin \left(\frac{3 \pi x}{L}\right) \cos (\theta), \\
w_{0}=W_{1}(t) \sin \left(\frac{\pi x}{L}\right) \sin (3 \theta)+W_{2}(t) \sin \left(\frac{3 \pi x}{L}\right) \sin (\theta), \\
\phi_{x}=\Psi_{x 1}(t) \cos \left(\frac{\pi x}{L}\right) \sin (3 \theta)+\Psi_{x 2}(t) \cos \left(\frac{3 \pi x}{L}\right) \sin (\theta), \\
\phi_{\theta}=\Psi_{\theta 1}(t) \sin \left(\frac{\pi x}{L}\right) \cos (3 \theta)+\Psi_{\theta 2}(t) \sin \left(\frac{3 \pi x}{L}\right) \cos (\theta),
\end{array}\right.
$$

where $W_{1}$ and $W_{2}(t)$ denote the time-dependent total amplitude; $U_{1}(t), U_{2}(t), V_{1}(t), V_{2}(t), \Psi_{x 1}(t), \Psi_{x 2}(t), \Psi_{\theta 1}(t)$, and $\Psi_{\theta 2}(t)$ denote the time-dependent amplitude functions.

Considering the evenly distributed periodic vibration load of the armature structure in engineering practice, to simulate the real loading conditions, the transverse uniformly distributed harmonic excitation is applied to the stiffened cylindrical shell. The uniformly distributed transverse harmonic excitation can be indicated as

$$
F=F_{1} \sin \left(\frac{\pi x}{L}\right) \cos (3 \theta)+F_{2} \sin \left(\frac{3 \pi x}{L}\right) \cos (\theta),
$$

where $F_{1}$ and $F_{2}$ represent the amplitudes of the transverse excitation.

Considering that the transverse nonlinear vibrations are the primary motion type for stiffened cylindrical shells, the inertia terms in equation (14) are removed in partial differential governing equations. By substituting equations (17) and (18) into (14) and applying the Galerkin discretization procedure, the equations in $u_{0}, v_{0}, \phi_{x}$, and $\phi_{\theta}$ directions in displacements equation are established simultaneously, in which the generalized coordinates $U_{1}(t), U_{2}(t), V_{1}(t), V_{2}(t)$, $\Psi_{x 1}(t), \Psi_{x 2}(t), \Psi_{\theta 1}(t)$, and $\Psi_{\theta 2}(t)$ can be expressed by the generalized coordinates $W_{1}$ and $W_{2}$ and brought into the $w_{0}$ direction, and the two-degree-of-freedom discrete ordinary differential governing equations of transverse motion for the stiffened cylindrical shell can be driven as

$$
\left\{\begin{array}{l}
\ddot{W}_{1}+\zeta_{12} \dot{W}_{1}+\omega_{1}^{2} W_{1}+\zeta_{14} W_{1}^{3}+\zeta_{15} W_{1} W_{2}^{2}+\zeta_{16} N_{1} W_{1} \cos (Q t)=\zeta_{17} F_{1} \cos (\Omega t) \\
\ddot{W}_{2}+\zeta_{22} \dot{W}_{2}+\omega_{2}^{2} W_{2}+\zeta_{24} W_{1}^{2} W_{2}+\zeta_{25} W_{2}^{3}+\zeta_{26} N_{1} W_{2} \cos (Q t)=\zeta_{27} F_{2} \cos (\Omega t)
\end{array}\right.
$$

where $W=\left[\begin{array}{ll}W_{1} & W_{2}\end{array}\right]^{T}$ represent the transverse displacements vectors; $\zeta_{i j}(i=1,2, j=2-7)$ represent the coefficients; $Q$ and $\Omega$ represent the excitation frequencies; point above variables represents the time derivative; $N_{1}$ represents the amplitude of in-plane excitation. Note that, even for the first two modes, the established governing equation also involves 
quadratic terms, cubic terms, and parametric and transverse excitations, which illustrates the high nonlinearity of transverse vibrations of stiffened cylindrical shells.

For the high-dimensional nonlinear dynamic systems, when there exists a special internal resonant relationship between two linear natural frequencies, the large amplitude nonlinear responses may suddenly happen owing to the modal interactions [58]. In this section, to reveal the nonlinear vibration behaviors of stiffened cylindrical shells, the perturbation analysis for the system's primary resonance is implemented with the multiscale method. By considering the case of $1: 2$ internal resonance and primary resonance-1/ 2 subharmonic parametric resonance, the resonant relations of stiffened cylindrical shells are given as follows:

$$
\begin{aligned}
2 \omega_{1} & =\omega-\varepsilon \sigma_{1}, \\
\omega_{2} & =\omega-\varepsilon \sigma_{2},
\end{aligned}
$$

where $\omega=Q=\Omega, \varepsilon$ is the small perturbation parameter, and $\sigma_{1}$ and $\sigma_{2}$ are the two detuning parameters.

To obtain the dimensionless ordinary differential governing equations for the stiffened cylindrical shell, the transformations of the variables and the parameters are introduced as follows:

$$
\begin{aligned}
& \tau=t \omega_{1}, \\
& W_{1}=f_{1} h \text {, } \\
& W_{2}=f_{2} h \text {, } \\
& \bar{\Omega}=\frac{\Omega}{\omega_{1}}, \\
& \bar{Q}=\frac{Q}{\omega_{1}}, \\
& \bar{\zeta}_{12}=\frac{\zeta_{12}}{\omega_{1}}, \\
& \bar{\zeta}_{14}=\frac{\zeta_{14} h^{2}}{\omega_{1}^{2}}, \\
& \bar{\zeta}_{15}=\frac{\zeta_{15} h^{2}}{\omega_{1}^{2}} \\
& \bar{\zeta}_{16}=\frac{\zeta_{16}}{\omega_{1}^{2}}, \\
& \bar{\zeta}_{17}=\frac{\zeta_{17}}{\omega_{1}^{2} h} \\
& \bar{\zeta}_{22}=\frac{\zeta_{22}}{\omega_{1}}, \\
& \bar{\zeta}_{24}=\frac{\zeta_{24} h^{2}}{\omega_{1}^{2}}
\end{aligned}
$$

$$
\begin{aligned}
\bar{\zeta}_{25} & =\frac{\zeta_{25} h^{2}}{\omega_{1}^{2}}, \\
\bar{\zeta}_{26} & =\frac{\zeta_{26}}{\omega_{1}^{2}}, \\
\bar{\zeta}_{27} & =\frac{\zeta_{27}}{\omega_{1}^{2} h}, \\
\bar{\omega}_{1} & =1, \\
\bar{\omega}_{2} & =\frac{\omega_{2}}{\omega_{1}}, \\
& =2,
\end{aligned}
$$

Substituting equation (21) into equation (19), the dimensionless equations are acquired as follows:

$$
\begin{aligned}
& \frac{\partial^{2} f_{1}}{\partial \tau^{2}}+\bar{\zeta}_{12} \frac{\partial f_{1}}{\partial \tau}+\bar{\omega}_{1}^{2} f_{1}+\bar{\zeta}_{14} f_{1}^{3}+\bar{\zeta}_{15} f_{1} f_{2}^{2}+\bar{\zeta}_{16} N_{1} \cos (\bar{Q} \tau) f_{1} \\
& =\bar{\zeta}_{17} F_{1} \cos (\bar{\Omega} \tau),
\end{aligned}
$$

$$
\begin{aligned}
& \frac{\partial^{2} f_{2}}{\partial \tau^{2}}+\bar{\zeta}_{22} \frac{\partial f_{2}}{\partial \tau}+\bar{\omega}_{2}^{2} f_{2}+\bar{\zeta}_{24} f_{1}^{2} f_{2}+\bar{\zeta}_{25} f_{2}^{3}+\bar{\zeta}_{26} N_{1} \cos (\bar{Q} \tau) f_{2} \\
& =\bar{\zeta}_{27} F_{2} \cos (\bar{\Omega} \tau) .
\end{aligned}
$$

To precisely obtain the uniform solutions of equations (22a) and (22b), the multiscale method [54] is employed in the following form:

$$
\begin{aligned}
f_{n}(t, \varepsilon)= & u_{n 0}\left(T_{0}, T_{1}, T_{2}\right)+\varepsilon u_{n 1}\left(T_{0}, T_{1}, T_{2}\right) \\
& +\varepsilon^{2} u_{n 2}\left(T_{0}, T_{1}, T_{2}\right)+\cdots, \quad(n=1,2),
\end{aligned}
$$

where time scale variables $T_{0}=\tau, T_{1}=\varepsilon \tau$, and $T_{2}=\varepsilon^{2} \tau$.

To express the equation conveniently, the time derivatives used in the multiscale method are given as follows:

$$
\begin{aligned}
\frac{\mathrm{d}}{\mathrm{d} t} & =\frac{\partial}{\partial T_{0}} \frac{\partial T_{0}}{\partial t}+\frac{\partial}{\partial T_{1}} \frac{\partial T_{1}}{\partial t}+\cdots=D_{0}+\varepsilon D_{1}+\cdots \\
\frac{\mathrm{d}^{2}}{\mathrm{~d} t^{2}} & =\left(D_{0}+\varepsilon D_{1}+\cdots\right)^{2} \\
& =D_{0}^{2}+2 \varepsilon D_{0} D_{1}+\cdots
\end{aligned}
$$

where the partial derivative operators $D_{0}=\partial / \partial T_{0}$ and $D_{1}=\partial / \partial T_{1}$.

Substituting equations (23) and (24b, 24a) into (22a) and (22b) and balancing the coefficients of the like power of $\varepsilon$ yield the following differential equations:

$$
\begin{aligned}
\varepsilon^{0} \text { order } D_{0}^{2} u_{10}+\omega_{1}^{2} u_{10} & =0, \\
D_{0}^{2} u_{20}+\omega_{2}^{2} u_{20} & =0,
\end{aligned}
$$




$$
\begin{aligned}
\varepsilon^{1} \operatorname{order} D_{0}^{2} u_{11}+\omega_{1}^{2} u_{11}= & \bar{\zeta}_{12} D_{0} u_{10}+2 D_{0} D_{1} u_{10}+\bar{\zeta}_{14}\left(u_{10}\right)^{3} \\
& +\bar{\zeta}_{15} u_{10}\left(u_{20}\right)^{2} \\
& +\bar{\zeta}_{16} N_{1} u_{10}\left(\frac{1}{2} e^{-i \bar{Q} T_{0}}+\frac{1}{2} e^{i \bar{Q} T_{0}}\right) \\
& -\bar{\zeta}_{17} F_{1}\left(\frac{1}{2} e^{-i T_{0} \bar{\Omega}}+\frac{1}{2} e^{i T_{0} \bar{\Omega}}\right) \\
D_{0}^{2} u_{21}+\omega_{2}^{2} u_{21}= & \bar{\zeta}_{22} D_{0} u_{20}+2 D_{0} D_{1} u_{20}+\bar{\zeta}_{24}\left(u_{10}\right)^{2} u_{2}^{(0)} \\
& +\bar{\zeta}_{25}\left(u_{20}\right)^{3} \\
& +\bar{\zeta}_{26} N_{1} u_{20}\left(\frac{1}{2} e^{-i \bar{Q} T_{0}}+\frac{1}{2} e^{i \bar{Q} T_{0}}\right) \\
& -\bar{\zeta}_{27} F_{2}\left(\frac{1}{2} e^{-i T_{0} \bar{\Omega}}+\frac{1}{2} e^{i T_{0} \bar{\Omega}}\right) .
\end{aligned}
$$

Since equations (25a) and (25b) are linear ordinary differential equations, the complex form solutions can be expressed as follows:

$$
\begin{aligned}
& u_{10}=A_{1}\left(T_{1}\right) e^{i \omega_{1} T_{0}}+e^{-i \omega_{1} T_{0}} \overline{A_{1}}\left(T_{1}\right), \\
& u_{20}=A_{2}\left(T_{1}\right) e^{i \omega_{2} T_{0}}+e^{-i \omega_{2} T_{0}} \overline{A_{2}}\left(T_{1}\right),
\end{aligned}
$$

where $\overline{A_{1}}$ and $\overline{A_{2}}$ are the complex conjugate of $A_{1}$ and $A_{2}$. Substituting equations (26a) and (26b) into (25c) and (25d) yield

$$
\begin{aligned}
D_{0}^{2} u_{11}+\omega_{1}^{2} u_{11}= & e^{i \omega_{1} T_{0}}\left(2 \zeta_{15} A_{1}\left(T_{1}\right) A_{2}\left(T_{1}\right) \overline{A_{2}}\left(T_{1}\right)+\frac{1}{2} \zeta_{16} N_{1} \overline{A_{1}}\left(T_{1}\right) e^{i \varepsilon \sigma_{1} T_{0}+i \varepsilon \sigma_{2} T_{0}}\right. \\
& \left.+3 \zeta_{14} A_{1}\left(T_{1}\right)^{2} \overline{A_{1}}\left(T_{1}\right)+i \zeta_{12} \omega_{1} A_{1}\left(T_{1}\right)+2 i D_{1} A_{1}\left(T_{1}\right) \omega_{1}\right)+\mathrm{cc}+\mathrm{NST}, \\
D_{0}^{2} u_{21}+\omega_{2}^{2} u_{21}= & e^{i \omega_{2} T_{0}}\left(2 \zeta_{24} A_{1}\left(T_{1}\right) A_{2}\left(T_{1}\right) \overline{A_{1}}\left(T_{1}\right)+3 \zeta_{25} A_{2}\left(T_{1}\right)^{2} \overline{A_{2}}\left(T_{1}\right)+i \zeta_{22} \omega_{2} A_{2}\left(T_{1}\right)\right. \\
& \left.-\frac{1}{2} \zeta_{27} F_{2} e^{i \varepsilon \sigma_{2} T_{0}}+2 i D_{1} A_{2}\left(T_{1}\right) \omega_{2}\right)+\mathrm{cc}+\mathrm{NST},
\end{aligned}
$$

where cc and NST denote the complex conjugate part on the right side of equation (27) and the terms that do not produce secular terms, respectively. Note that the variable symbol $\bar{\zeta}$ is rewritten as $\zeta$ to facilitate the following formula derivation.

To write the modulation equations in polar coordinates, the amplitude functions are set as functions $A_{1}$ and $A_{2}$, which can be denoted in the exponential form as follows:

$$
\begin{aligned}
& A_{1}\left(T_{1}\right)=\frac{1}{2} a_{1} e^{i \phi_{1}}, \\
& A_{2}\left(T_{1}\right)=\frac{1}{2} a_{2} e^{i \phi_{2}} .
\end{aligned}
$$

Substituting equations (28a) and (28b) into (27a) and (27b) and separating the real and imaginary parts, fourdimensional averaged equations in the polar form are obtained:

$$
\begin{aligned}
& \dot{a}_{1}=\frac{1}{4} \zeta_{16} a_{1} N_{1} \sin \left(\phi_{1}\right)+\frac{1}{2} \zeta_{12} a_{1} \omega_{1}, \\
& \dot{a}_{2}=\frac{1}{2} \zeta_{22} a_{2} \omega_{2}-\frac{1}{2} \zeta_{27} F_{2} \sin \left(\phi_{2}\right), \\
& \dot{\phi}_{1}=\frac{1}{4} \zeta_{15} a_{1} a_{2}^{2}+\frac{1}{4} \zeta_{16} a_{1} N_{1} \cos \left(\phi_{1}\right)-\frac{1}{2} a_{1} \omega_{1}\left(\sigma_{1}+\sigma_{2}\right)+\frac{3 \zeta_{14} a_{1}^{3}}{8},
\end{aligned}
$$

$$
\dot{\phi}_{2}=\frac{1}{4} \zeta_{24} a_{1}^{2} a_{2}+\frac{3 \zeta_{25} a_{2}^{3}}{8}-\frac{1}{2} \zeta_{27} F_{2} \cos \left(\phi_{2}\right)-a_{2}\left(T_{1}\right) \sigma_{2} \omega_{2} .
$$

Ultimately, to obtain the numerical solutions effectively, the complex partial differential kinetic equations (i.e., equation (14)) are simplified into simple four-dimensional average equations (i.e., equation (29)).

\section{Analysis of Resonant Responses}

In this section, based on the established four-dimensional averaged equations (equations (29a)-(29d)) in the polar form, by considering the electromagnetic excitations, transverse excitations, and in-plane excitations, the amplitude-frequency characteristics of stiffened cylindrical shells are investigated in this section. It should be noted that the established four-dimensional averaged equations are derived and solved on MATHEMATICA and MATLAB, respectively. The basic parameters of the stiffened cylindrical shell are set as follows: elastic modulus $E=2.1 \times 10^{11} \mathrm{~Pa}$, density $\rho=7800 \mathrm{~kg} / \mathrm{m}^{3}$, Poisson's ratio $\nu=0.3$, conductivity $\sigma=2.3 \times 10^{6}(\Omega \cdot \mathrm{m})^{-1}, L=0.485 \mathrm{~m}, R=0.1665 \mathrm{~m}, h=0.007 \mathrm{~m}$, and in-plane and transverse excitation frequencies are $Q=\Omega=150 \mathrm{~Hz}$ [26]. Moreover, by setting the left parts of equations (equations (29a)-(29d)) to equal zero, the effects of different parameters on the resonant responses of the stiffened cylindrical shell are studied, and the nonlinear 
dynamic responses are described in amplitude-frequency curves under the cases of the primary resonance- $1 / 2$ subharmonic parametric resonance and $1: 2$ internal resonance, as shown in Figures 4-7. Therein, it is worth noting that the dotted line represents the unstable region, the solid line indicates the stable area, and the red dot represents the limit point in these amplitude-frequency curves.

Figure 4 shows the influence of different transverse excitation amplitude on the amplitude-frequency curve. The simulation results reveal that the amplitude of the first-order mode is larger than that of the second-order mode. Both the first two modes make left bending formants, so it can be judged that the system makes nonlinear softening characteristics. Moreover, with the increase of transverse excitation amplitude $F_{2}$, the amplitudes and resonant interval become larger and the unstable region increases gradually. These changes indicate that the stronger resonance responses are excited since more energy is absorbed with the increase of the transverse excitation. Furthermore, the resonance regions of the first-order modes are consistent with that of the second-order modes, which illustrates that the resonance of the first-order modes is caused by the main resonance phenomenon of the second-order modes. Besides, the bending of the amplitude-frequency curves also leads to the multiple amplitudes and the jump phenomena. Herein, the multiple amplitudes mean that there are many possible responses in stiffened cylindrical shells, and the actual responses are determined by the initial conditions; the jump phenomenon in stiffened cylindrical shells means that the amplitude changes abruptly.

To investigate the effects of in-plane excitation amplitude $N_{1}$ on the amplitude-frequency response, the amplitude variation of the modes is analyzed, and the results are shown in Figure 5. In Figure 5, the upper and lower four curves represent the amplitude-frequency curve of the first two modes when $N_{1}=3.2 \times 10^{7} \mathrm{~N} / \mathrm{m}^{2}, \quad N_{1}=3.5 \times 10^{7} \mathrm{~N} / \mathrm{m}^{2}$, $N_{1}=3.8 \times 10^{7} \mathrm{~N} / \mathrm{m}^{2}$, and $N_{1}=4.1 \times 10^{7} \mathrm{~N} / \mathrm{m}^{2}$, respectively. It can be seen from the figure that, with the increase of tuning parameter $\sigma_{1}$, there will be a jump of amplitude in the first two modal amplitudes in an unstable region. Moreover, we also find that the first-order and the second-order modal limit points are $\sigma_{1}=0.2021$ and $\sigma_{1}=0.2818$ when $N_{1}=3.2 \times 10^{7} \mathrm{~N} / \mathrm{m}^{2} ; \quad \sigma_{1}=0.2402$ and $\sigma_{1}=0.3194$ when $N_{1}=3.5 \times 10^{7} \mathrm{~N} / \mathrm{m}^{2} ; \quad \sigma_{1}=0.2769$ and $\sigma_{1}=0.3570$ when $N_{1}=3.8 \times 10^{7} \mathrm{~N} / \mathrm{m}^{2} ; \quad \sigma_{1}=0.3145$ and $\sigma_{1}=0.3945$ when $N_{1}=4.1 \times 10^{7} \mathrm{~N} / \mathrm{m}^{2}$. The results show that, with the increase of in-plane excitation amplitude $N_{1}$, the unstable region gradually moves to the right, which means that the natural frequency of the system is increasing. Besides, with the increase of $N_{1}$, the ordinates of limit points of the first-order modes move upward, while the ordinates of limit points of the second-order modes remain unchanged, indicating that the increase of $N_{1}$ increases the energy of the first-order modes but has little effect on the amplitude of the secondorder modes. These results illustrate that the subharmonic parametric resonance which is forced by axial in-plane excitation occurs in the first modes.

To verify the effects of electromagnetic intensities $B$ on the amplitude-frequency response of the system, the

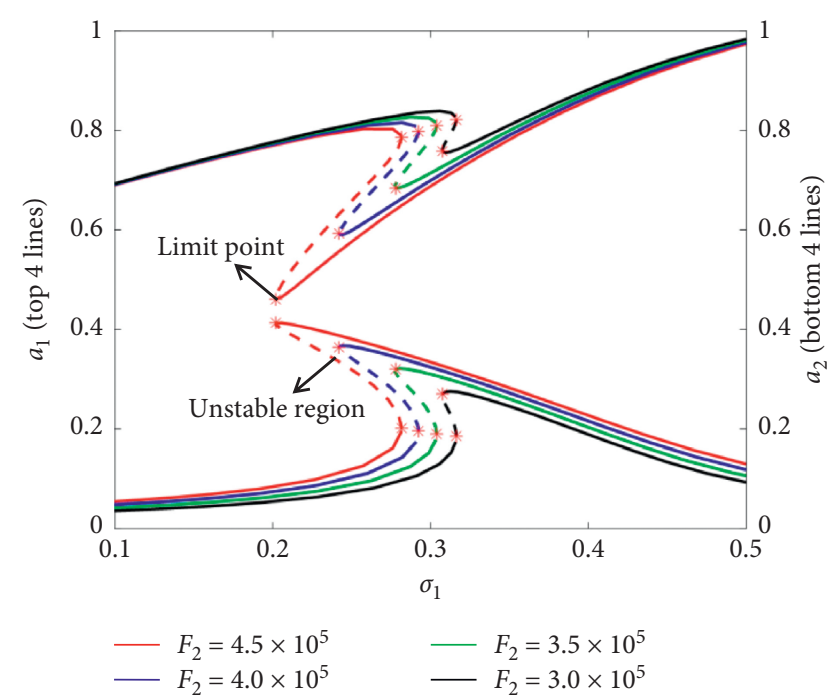

FIGURE 4: Amplitude-frequency curves with different transverse excitations.

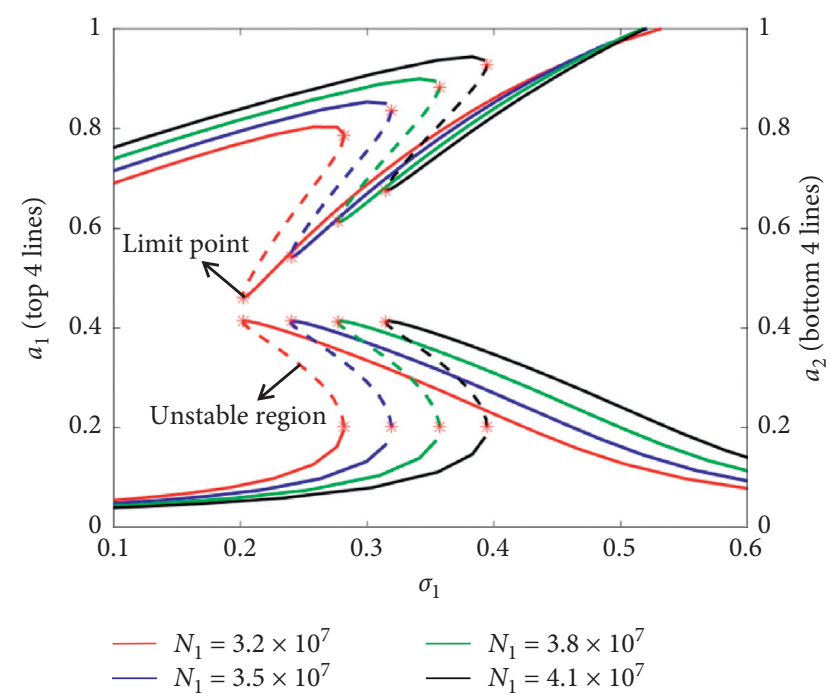

Figure 5: Amplitude-frequency curves with different in-plane excitations.

amplitude changes of the modes under different electromagnetic intensities are analyzed. By setting transverse excitation amplitude $F_{2}=4.5 \times 10^{5} \mathrm{~N} / \mathrm{m}^{2}$, in-plane excitation amplitude $N_{1}=3.2 \times 10^{7} \mathrm{~N} / \mathrm{m}^{2}$, and the amplitude-frequency curves are drawn in Figure 6. Note that the upper and lower four curves represent the amplitude-frequency response curve of the first two modes when $B=(18-21) \mathrm{T}$, respectively. From the figure, we find that limit points of first two modal tuning parameters are $\sigma_{1}=0.2021$ and $\sigma_{1}=0.2818$ when $B=18 \mathrm{~T} ; \sigma_{1}=0.2402$ and $\sigma_{1}=0.3194$ when $B=19 \mathrm{~T}$; $\sigma_{1}=0.2769$ and $\sigma_{1}=0.3570$ when $B=20 \mathrm{~T} ; \sigma_{1}=0.3145$ and $\sigma_{1}=0.3945$ when $B=21 \mathrm{~T}$. Besides, with the increase of the electromagnetic intensity, the energy of the first two modes is decreasing gradually. Moreover, when passing through the resonance frequency, a certain degree of the mutation of the first two modes is discovered. 


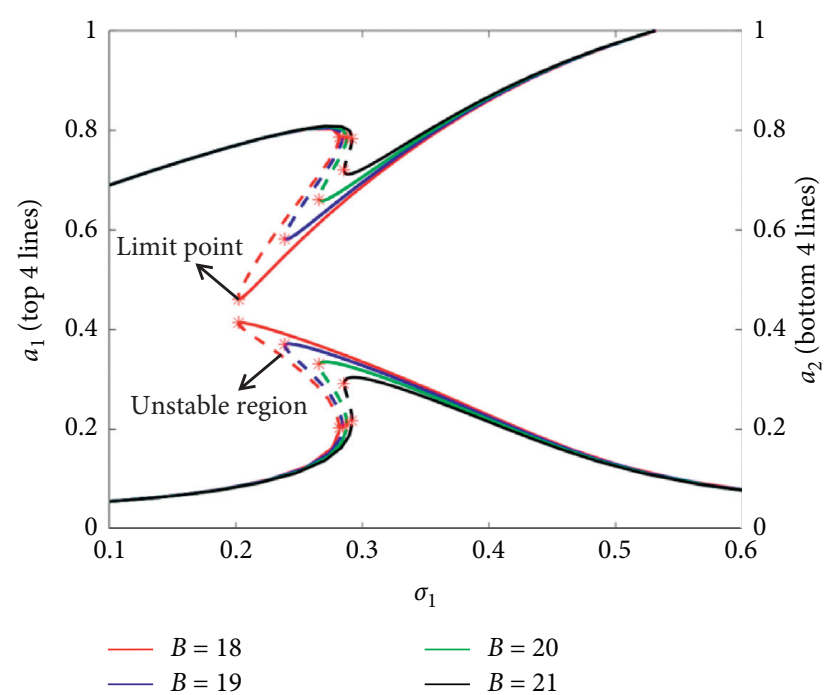

FIgURE 6: Amplitude-frequency curves with different electromagnetic intensities.

Figure 7 shows the effect of the small tuning parameter $\sigma_{2}$ on the amplitude-frequency responses of the system, in which $\sigma_{2}$ is used to adjust the second-order frequency. The upper and lower four curves represent the amplitude-frequency curve of the first two modes when $\sigma_{2}=0 ; \sigma_{2}=0.05$ and $\sigma_{2}=0.10 ; \sigma_{2}=0.15$, respectively. The two limit points of the first two modal tuning parameters are $\sigma_{1}=0.2021$ and $\sigma_{1}=0.2818$ when $\sigma_{2}=0 ; \sigma_{1}=0.2658$ and $\sigma_{1}=0.3328$ when $\sigma_{2}=0.05 ; \sigma_{1}=0.3220$ and $\sigma_{1}=0.3784$ when $\sigma_{2}=0.10$; $\sigma_{1}=0.3721$ and $\sigma_{1}=0.4193$ when $\sigma_{2}=0.15$. It can be found that when increasing the small tuning parameter $\sigma_{2}$, the unstable region gradually shifts to the right. Meanwhile, with the increase of $\sigma_{2}$, the amplitudes and resonant intervals become greater in amplitude-frequency curves. Besides, the amplitude of the first-order modes uniformly moves up and the ordinates of limit points move down, while the ordinates of limit points of the second-order modes remain unchanged. It is indicated that, with the increase of small tuning parameter $\sigma_{2}$, the energy of the first-order modes will increase obviously, while the amplitude of the second-order modes will not be affected basically.

\section{Numerical Simulation of Periodic and Chaotic Dynamics}

In this section, with consideration of the influence of stiffener number, stiffener size, and aspect ratio, the periodic and the chaotic motions of stiffened cylindrical shells in the case of 1 : 2 internal resonance and primary resonance- $1 / 2$ subharmonic parametric resonance are studied in the following four cases. Therein, Case 1 is used to verify the feasibility of the proposed method, and its solutions are regarded as the benchmark results of the following cases; Cases 2-4 are applied to reveal the influence of stiffeners on the nonlinear vibration behaviors by adjusting the parameters (i.e., stiffener number, stiffener height, and aspect ratio). The change of parameters in Cases 1-4 is illustrated in Table 1.

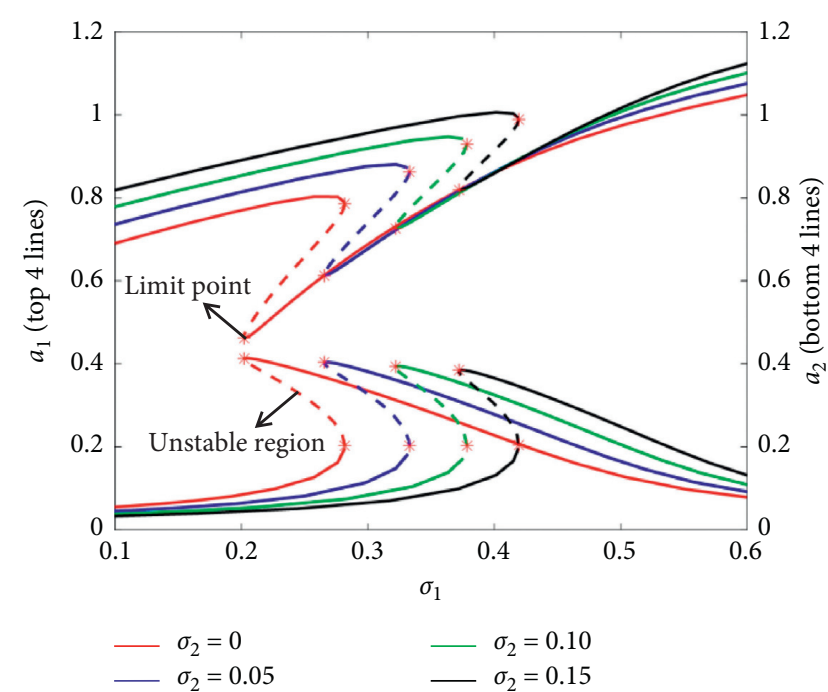

FIgURE 7: Amplitude-frequency curves with different small tuning parameters.

TABle 1: The change of parameters in Cases 1-4.

\begin{tabular}{lcccc}
\hline Parameters & $\begin{array}{c}\text { Stiffener } \\
\text { number }\end{array}$ & $\begin{array}{c}\text { Stiffener height } \\
(\mathrm{m})\end{array}$ & $\begin{array}{c}\text { Aspect } \\
\text { ratio }\end{array}$ & Others \\
\hline Case 1 & 5 & 0.003 & 2 & Same \\
Case 2 & 10 & 0.003 & 2 & Same \\
Case 3 & 5 & 0.006 & 2 & Same \\
Case 4 & 5 & 0.003 & 3.5 & Same \\
\hline
\end{tabular}

5.1. Case 1. To describe the nonlinear vibration responses of stiffened cylindrical shells, the basic parameters of the stiffened cylindrical shell are set as follows: elastic modulus $E=2.1 \times 10^{11} \mathrm{~Pa}$, density $\rho=7800 \mathrm{~kg} / \mathrm{m}^{3}$, Poisson's ratio $\nu=0.3$, conductivity $\sigma=2.3 \times 10^{6}(\Omega \cdot \mathrm{m})^{-1}$, shell length $L=0.485 \mathrm{~m}$, shell middle surface radius $R=0.1665 \mathrm{~m}$, shell thickness $h=0.007 \mathrm{~m}$, stiffener height $h_{s}=0.003 \mathrm{~m}$, stiffener spacing $b_{s}=0.097 \mathrm{~m}$, stiffener thickness $d_{s}=0.015 \mathrm{~m}$, and electromagnetic intensity $B=15 \mathrm{~T}$. Considering the transverse excitation amplitude $F_{1}=F_{2}=1 \times 10^{4} \mathrm{~N}$ and in-plane and transverse excitation frequencies $Q=\Omega=150 \mathrm{~Hz}$, the periodic and chaotic motions of the stiffened cylindrical shells are analyzed by adopting the fourth-order Runge-Kutta approach. Note that the in-plane excitation amplitude $N_{1}$ is assumed as a variable, while the basic parameters remained as the fixed values in numerical analysis.

To globally view the nonlinear vibration behaviors of the stiffened cylindrical shell, the bifurcation diagrams of the first two modes are depicted in Figure 8. Herein, the horizontal axis represents the in-plane excitation amplitude $N_{1}$ and the vertical axis represents the value of the first two modal displacements $W_{1}$ and $W_{2}$. Figure 8 shows that, with the increase of in-plane excitation amplitude, there exist periodic and chaotic motions in two main different regions. As $N_{1}$ increased past $N_{1}=5.92 \times 10^{7}$, the system undergoes changes from periodic motions to chaotic motions. The variation results are displayed in Figures 9 and 10. In these 


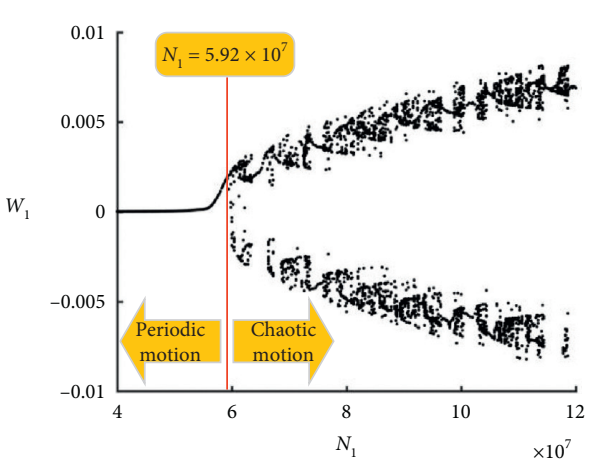

(a)

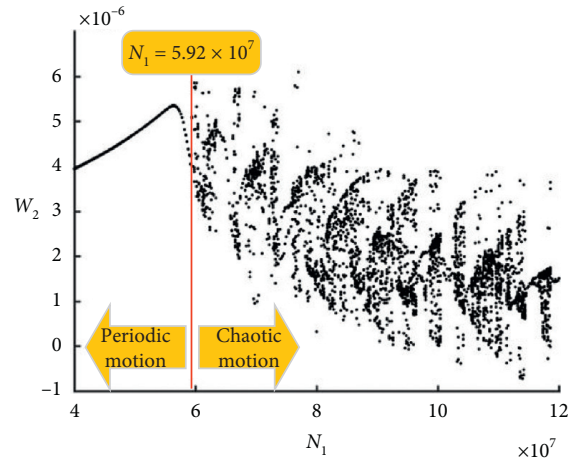

(b)

FIGURE 8: Bifurcation diagrams of stiffened cylindrical shell. (a) First-order bifurcation diagram. (b) Second-order bifurcation diagram.

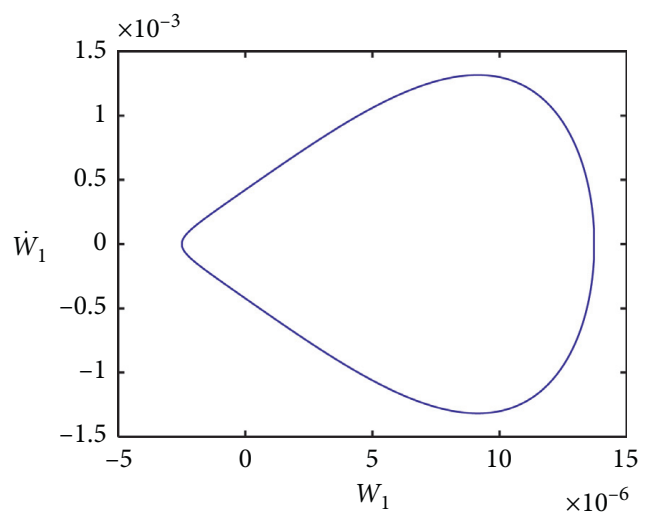

(a)

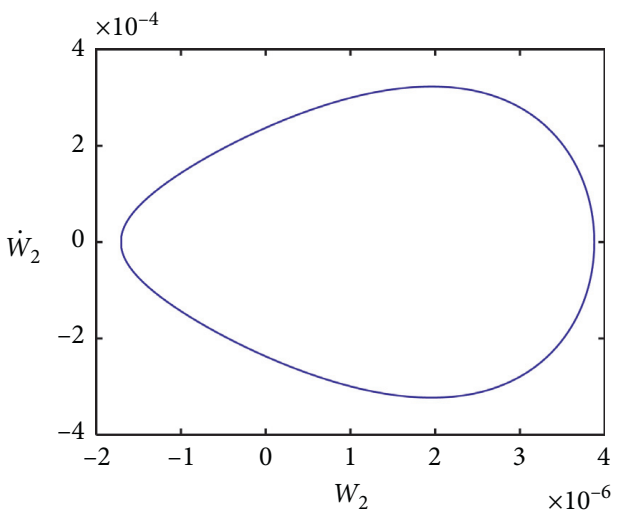

(c)

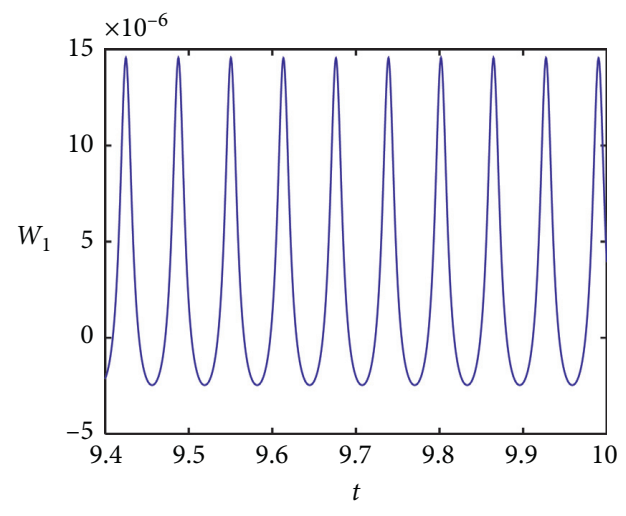

(b)

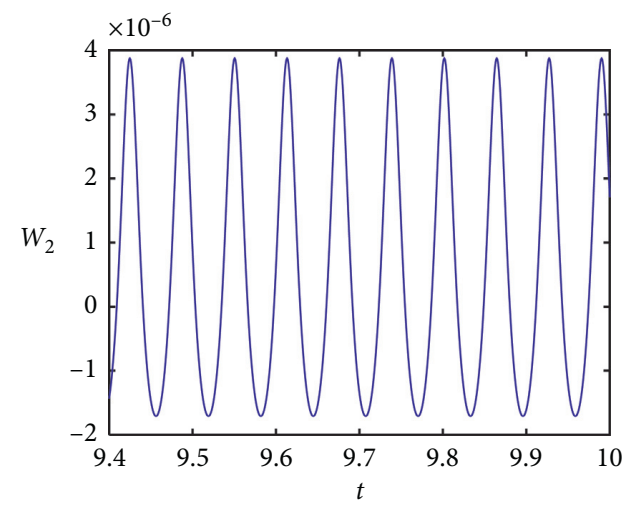

(d)

FIgURE 9: Continued. 


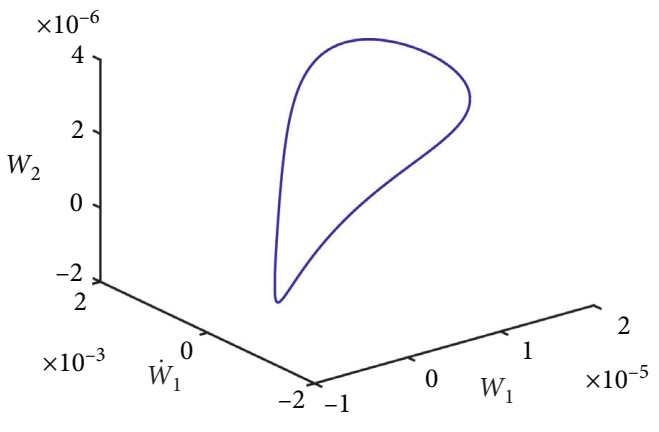

(e)

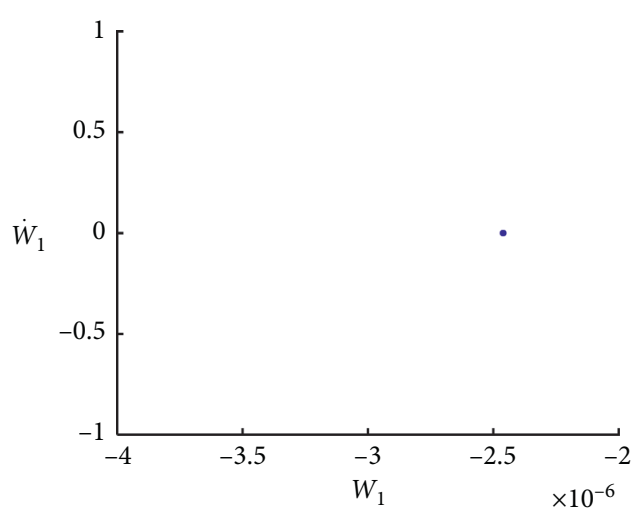

(f)

FIgUre 9: Periodic motion of the stiffened cylindrical shell when $N_{1}=4 \times 10^{7} \mathrm{~N} / \mathrm{m}^{2}$. (a) Phase portraits on the plane $\left(W_{1}\right.$, $\left.\dot{W}_{1}\right)$. (b) Waveform on the plane $\left(t, W_{1}\right)$. (c) Phase portraits on the plane $\left(W_{2}, \dot{W}_{2}\right)$. (d) Waveform on the plane $\left(t, W_{2}\right)$. (e) Three-dimensional phase portrait $\left(W_{1}, \dot{W}_{1}, W_{2}\right)$. (f) Poincare map on plane $\left(W_{1}, \dot{W}_{1}\right)$.

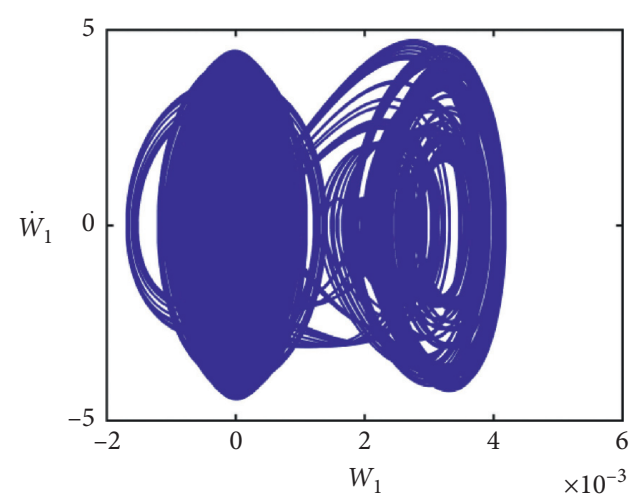

(a)

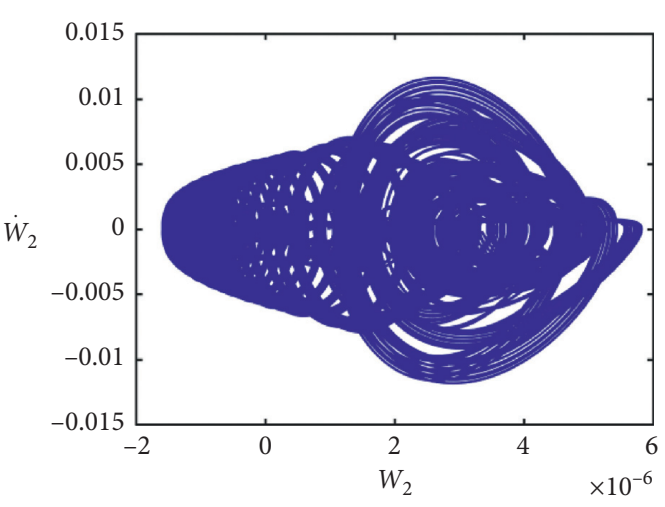

(c)

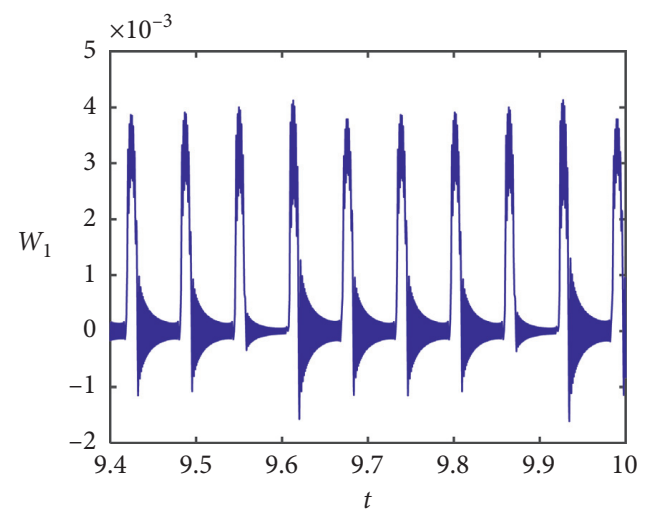

(b)

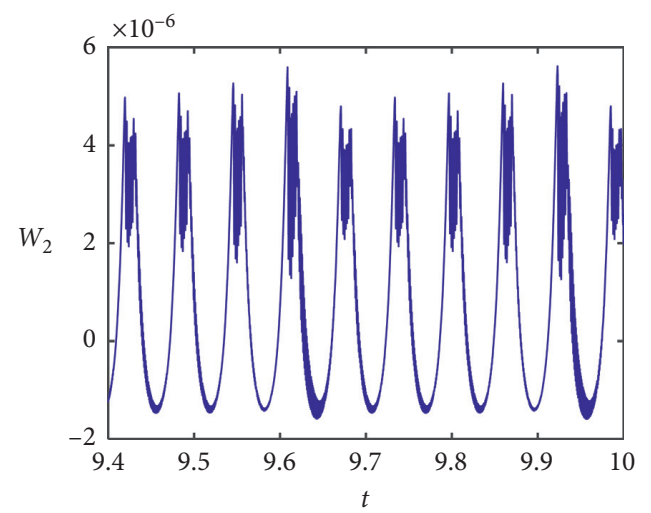

(d)

Figure 10: Continued. 


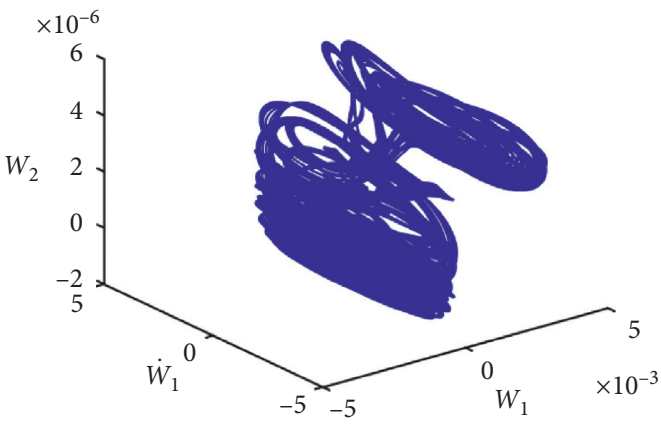

(e)

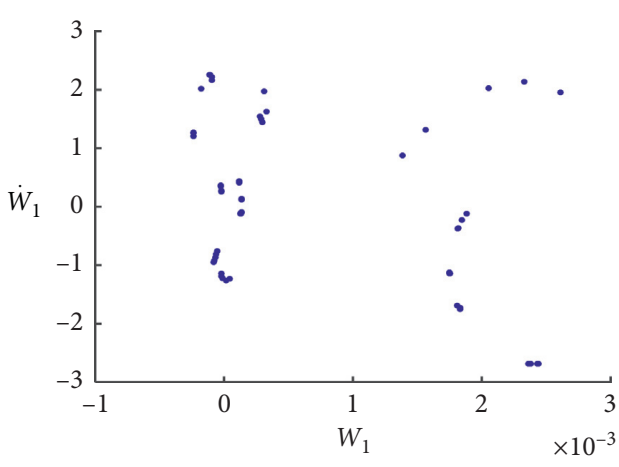

(f)

Figure 10: Chaotic motion of the stiffened cylindrical shell when $N_{1}=7 \times 10^{7} \mathrm{~N} / \mathrm{m}^{2}$. (a) Phase portraits on the plane $\left(W_{1}, \dot{W}_{1}\right)$. (b) Waveform on the plane $\left(t, W_{1}\right)$. (c) Phase portraits on the plane $\left(W_{2}, \dot{W}_{2}\right)$. (d) Waveform on the plane $\left(t, W_{2}\right)$. (e) Three-dimensional phase portrait $\left(W_{1}, \dot{W}_{1}, W_{2}\right)$. (f) Poincare map on plane $\left(W_{1}, \dot{W}_{1}\right)$.

figures, (a) and (c) represent the phase portraits on the planes $\left(W_{1}, \dot{W}_{1}\right)$ and $\left(W_{2}, \dot{W}_{2}\right)$, respectively; (b) and (d) depict the waveforms on the planes $\left(t, W_{1}\right)$ and $\left(t, W_{2}\right)$, respectively; (e) and (f) represent three-dimensional phase portrait in the space of $\left(W_{1}, \dot{W}_{1}, W_{2}\right)$ and the Poincare map on the plane $\left(W_{1}, \dot{W}_{1}\right)$, respectively; Figure $9(\mathrm{f})$ shows one point in Poincare maps, which indicates that the system makes the periodic motions when $N_{1}=4 \times 10^{7} \mathrm{~N} / \mathrm{m}^{2}$. Under the circumstances of $N_{1}=7 \times 10^{7} \mathrm{~N} / \mathrm{m}^{2}$, we observe that each excitation cycle produces different motion states in Figures 10(a)-10(e) and the Poincare map shows a lot of irregular points in Figure 10(f); the dynamic phenomenon illustrates that the system makes the chaotic motion behavior. Note that $N_{1}$ indicates the in-plane excitation amplitude, while $W_{1}$ and $W_{2}$ represent the first and second modal deformation, respectively.

5.2. Case 2. The flexural, compressive, or bearing capacity of stiffened cylindrical shells is seriously affected by the geometric configurations of stiffeners. To improve the mechanical performance (such as strength and stiffness) and increase the structural reliability of stiffened cylindrical shells, the effects of stiffener number on nonlinear responses under electromagnetic environment are investigated in this subsection. The geometric parameters of the stiffened cylindrical shell are set as follows: shell length $L=0.485 \mathrm{~m}$, shell middle surface radius $R=0.1665 \mathrm{~m}$, shell thickness $h=0.007 \mathrm{~m}$, stiffener number $n=10$, stiffener height $h_{s}=0.003 \mathrm{~m}$, stiffener spacing $b_{s}=0.0485 \mathrm{~m}$, and stiffener thickness $d_{s}=0.015 \mathrm{~m}$.

The bifurcation diagrams of the first two modes are described in Figure 11. From Figure 11, it can be seen that the shell's motions change from periodic motions to complex chaotic motions when $N_{1}=8.155 \times 10^{7} \mathrm{~N} / \mathrm{m}^{2}$. Figure 12 describes the nonlinear dynamic responses when in-plane excitation amplitude $N_{1}=6.5 \times 10^{7} \mathrm{~N} / \mathrm{m}^{2}$. At this time, there is only one fixed point in the Poincare map, which indicates that the stiffened cylindrical shell is in single period motion. When $N_{1}=9.5 \times 10^{7} \mathrm{~N} / \mathrm{m}^{2}$, the nonlinear dynamic responses of the stiffened cylindrical shell are drawn in Figure 13: there are infinite irregular points in the Poincare map and the motion in the time history diagram is also irregular; this phenomenon indicates that the stiffened cylindrical shell makes chaotic motion behavior. Compared with Figures 8 and 11, we observed that when increasing stiffener number $n$ from 5 to 10 , the critical value of $N_{1}$ will increase from $N_{1}=5.92 \times 10^{7} \mathrm{~N} / \mathrm{m}^{2}$ to $8.155 \times 10^{7} \mathrm{~N} / \mathrm{m}^{2}$, which illustrates that the chaotic motion of stiffened cylindrical shells can be effectively restrained by increasing the stiffener number.

5.3. Case 3. To verify the effects of various stiffening configurations on the nonlinear dynamic responses, the periodic and chaotic motions of the modes for stiffened cylindrical shells are analyzed. The geometries of the stiffened cylindrical shell are chosen as follows: shell length $L=0.485 \mathrm{~m}$, shell middle surface radius $R=0.1665 \mathrm{~m}$, shell thickness $h=0.007 \mathrm{~m}$, stiffener height $h_{s}=0.006 \mathrm{~m}$, stiffener spacing $b_{s}=0.097 \mathrm{~m}$, stiffener thickness $d_{s}=0.015 \mathrm{~m}$, and electromagnetic intensity $B=15 \mathrm{~T}$. The bifurcation diagrams when stiffener height $h_{s}=0.006 \mathrm{~m}$ are drawn in Figure 14. From Figure 14, it can be seen that the shell's motions change from periodicities to complex chaotic motions with the increase of in-plane excitation amplitude $N_{1}$. And the chaotic motion of the system would emerge when $N_{1}=9.45 \times 10^{7} \mathrm{~N} / \mathrm{m}^{2}$. Moreover, the nonlinear dynamic responses of $N_{1}=6.5 \times 10^{7} \mathrm{~N} / \mathrm{m}^{2}$ and $9.5 \times 10^{7} \mathrm{~N} / \mathrm{m}^{2}$ are shown in Figures 15 and 16 , respectively.

From the comparison of Figures 8 and 14, we find that the critical value of $N_{1}$ increases from $5.92 \times 10^{7} \mathrm{~N} / \mathrm{m}^{2}$ to $9.45 \times 10^{7} \mathrm{~N} / \mathrm{m}^{2}$ as the stiffener height increases from $0.003 \mathrm{~m}$ to $0.006 \mathrm{~m}$. It can be seen that the higher stiffener height can effectively suppress the chaotic bifurcation behavior of the stiffened cylindrical shell.

5.4. Case 4. Considering that different aspect ratios of stiffened cylindrical shells in different operating environments will show vastly different response characteristics, we further explore the influence of aspect ratio on dynamic responses of stiffened cylindrical shells in this subsection. By setting the aspect ratio as $L / R=3.5$, the bifurcation diagrams 


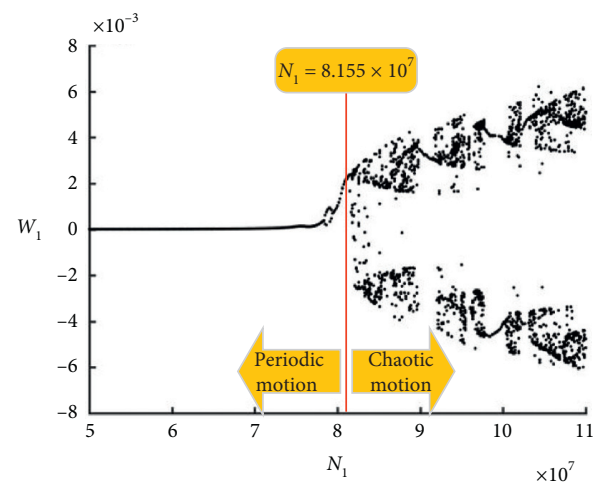

(a)

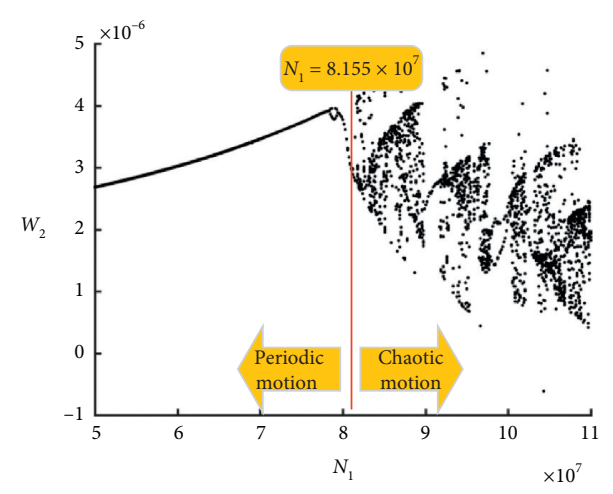

(b)

FIGURE 11: Bifurcation diagrams of stiffened cylindrical shell with stiffener number $n=10$. (a) First-order bifurcation diagram. (b) Secondorder bifurcation diagram.

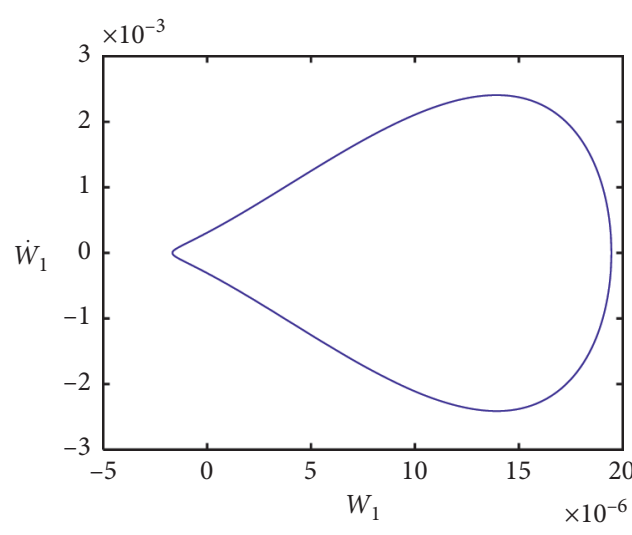

(a)

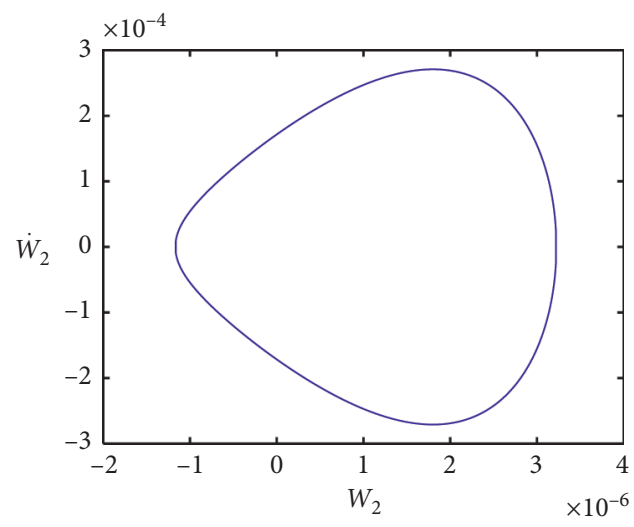

(c)

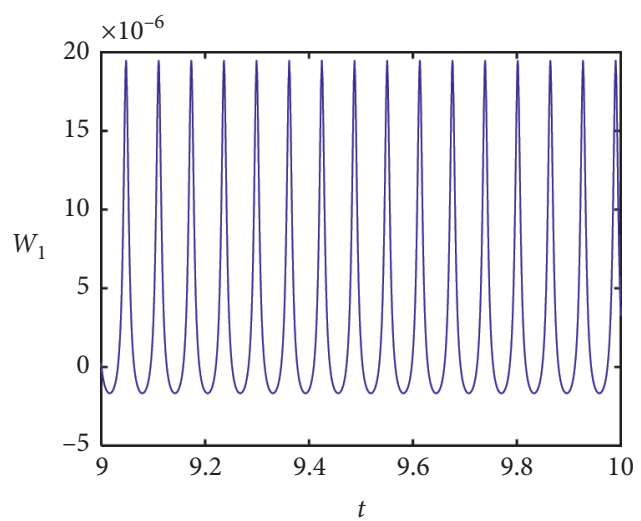

(b)

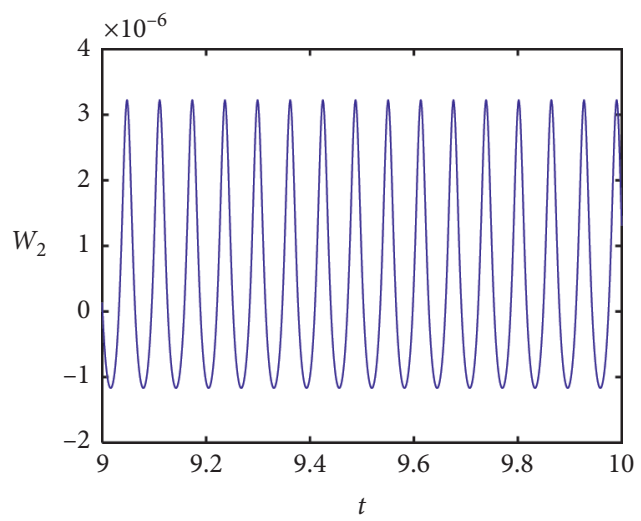

(d)

Figure 12: Continued. 


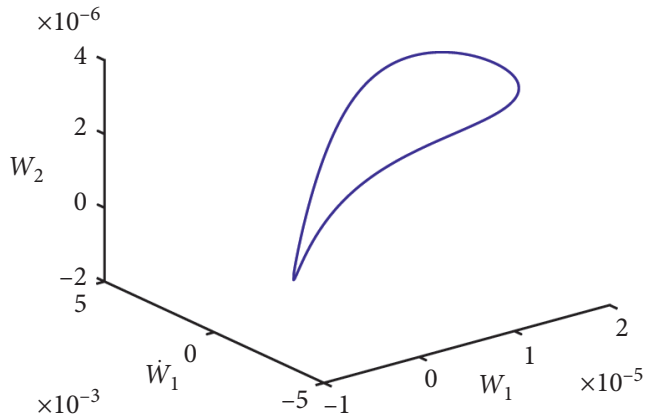

(e)

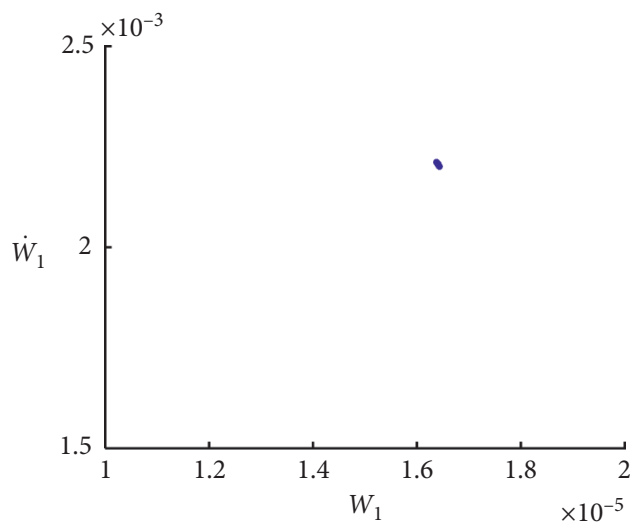

(f)

Figure 12: Periodic motion of the stiffened cylindrical shell when $N_{1}=6.5 \times 107 \mathrm{~N} / \mathrm{m}^{2}$. (a) Phase portraits on the plane $\left(W_{1}, \dot{W}_{1}\right) .(\mathrm{b})$ Waveform on the plane $\left(t, W_{1}\right)$. (c) Phase portraits on the plane $\left(W_{2}, \dot{W}_{2}\right)$. (d) Waveform on the plane $\left(t, W_{2}\right)$. (e) Three-dimensional phase portrait $\left(W_{1}, \dot{W}_{1}, W_{2}\right)$. (f) Poincare map on plane $\left(W_{1}, \dot{W}_{1}\right)$.

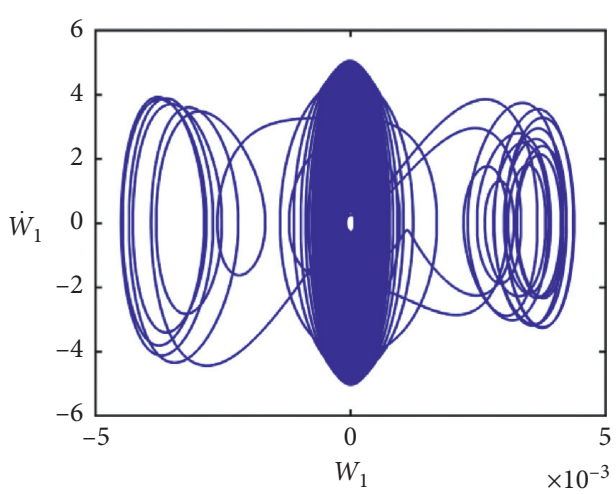

(a)

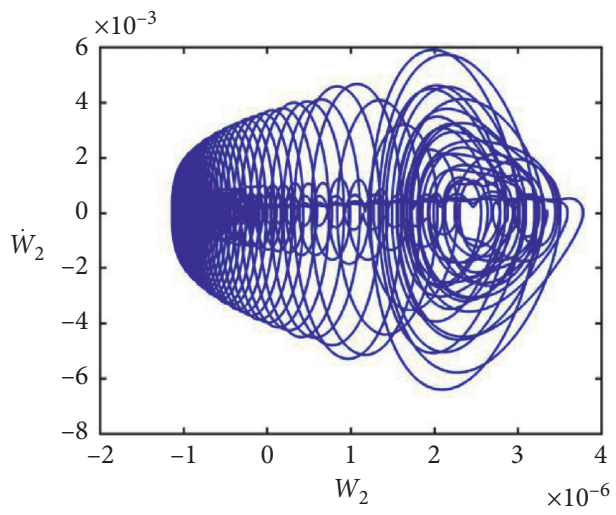

(c)

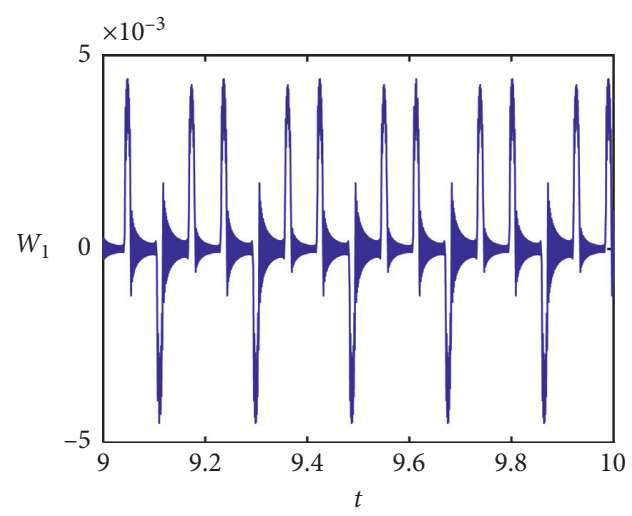

(b)

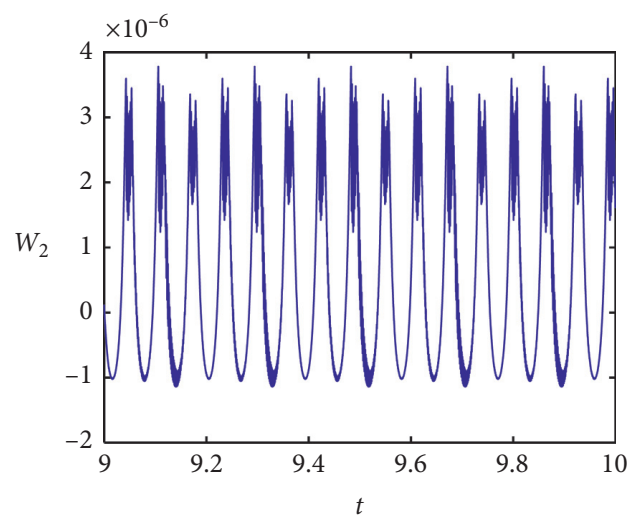

(d)

FIgURE 13: Continued. 


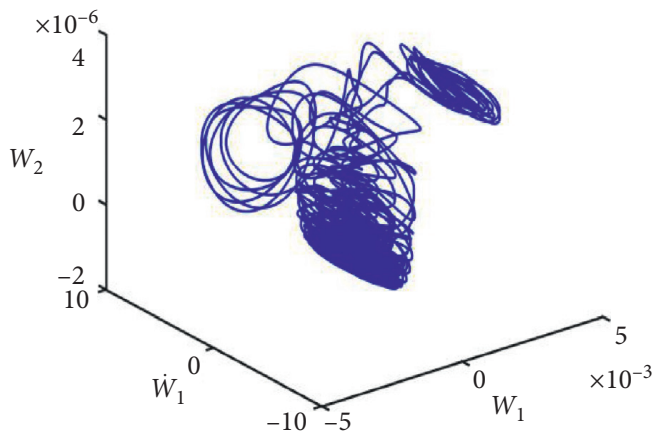

(e)

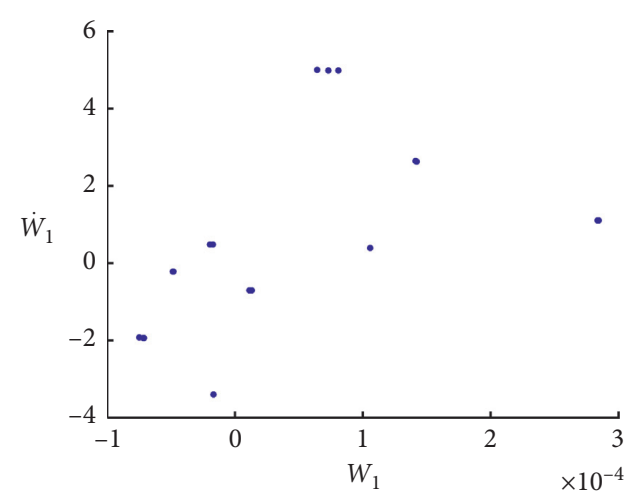

(f)

Figure 13: Chaotic motion of the stiffened cylindrical shell when $N_{1}=9.5 \times 10^{7} \mathrm{~N} / \mathrm{m}^{2}$. (a) Phase portraits on the plane $\left(W_{1}, \dot{W}_{1}\right)$. (b) Waveform on the plane $\left(t, W_{1}\right)$. (c) Phase portraits on the plane $\left(W_{2}, \dot{W}_{2}\right)$. (d) Waveform on the plane $\left(t, W_{2}\right)$. (e) Three-dimensional phase portrait $\left(W_{1}, \dot{W}_{1}, W_{2}\right)$. (f) Poincare map on plane $\left(W_{1}, \dot{W}_{1}\right)$.

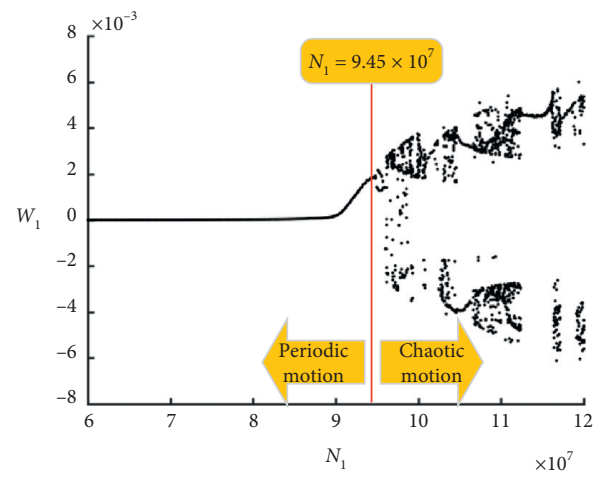

(a)

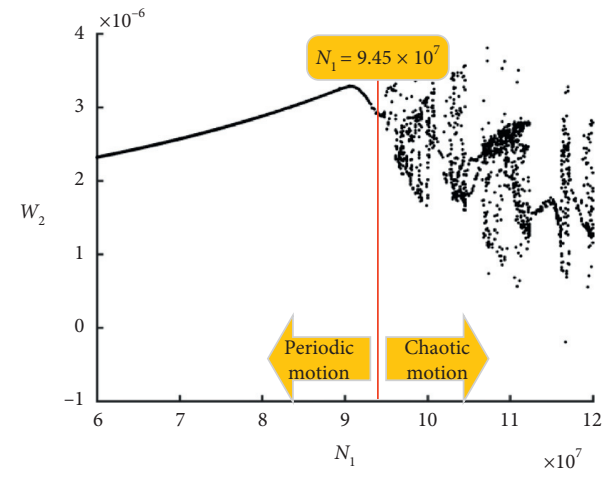

(b)

FIgURE 14: Bifurcation diagrams of stiffened cylindrical shell with stiffener height $h_{s}=0.006 \mathrm{~m}$. (a) First-order bifurcation diagram. (b) Second-order bifurcation diagram.

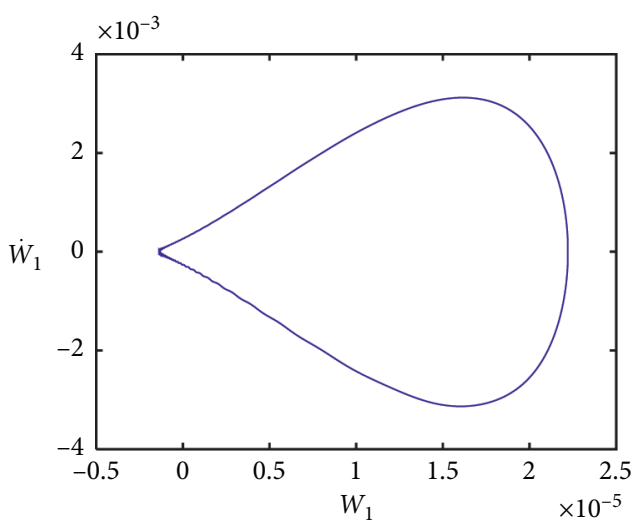

(a)

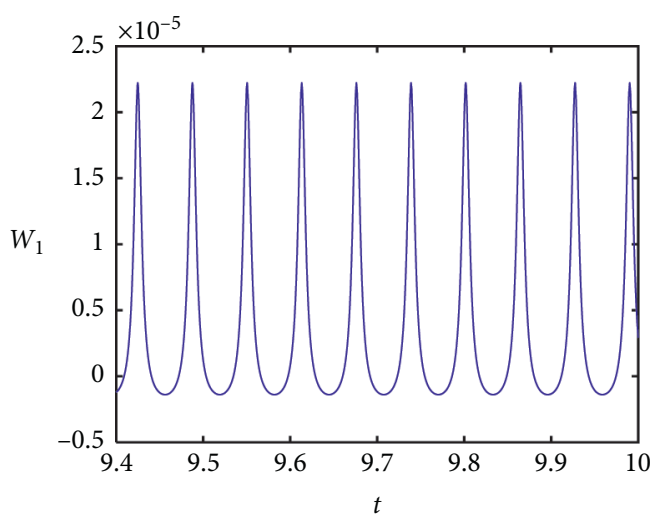

(b)

Figure 15: Continued. 


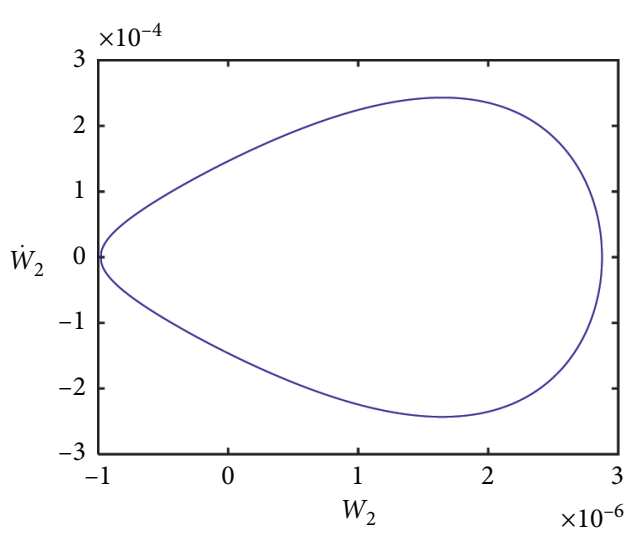

(c)

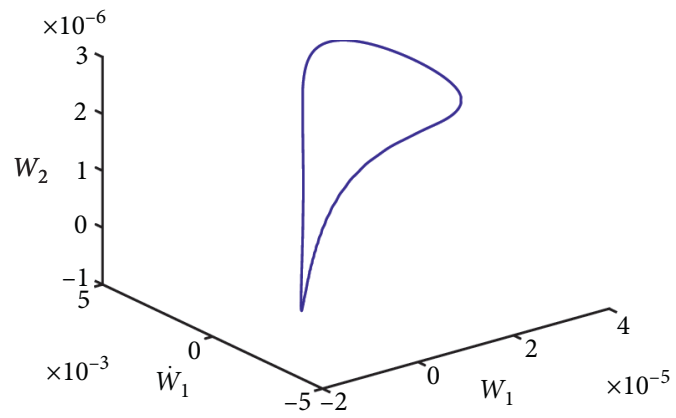

(e)

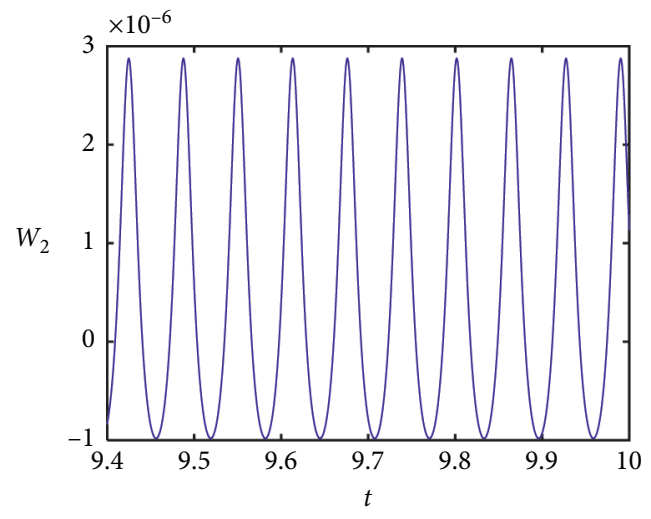

(d)

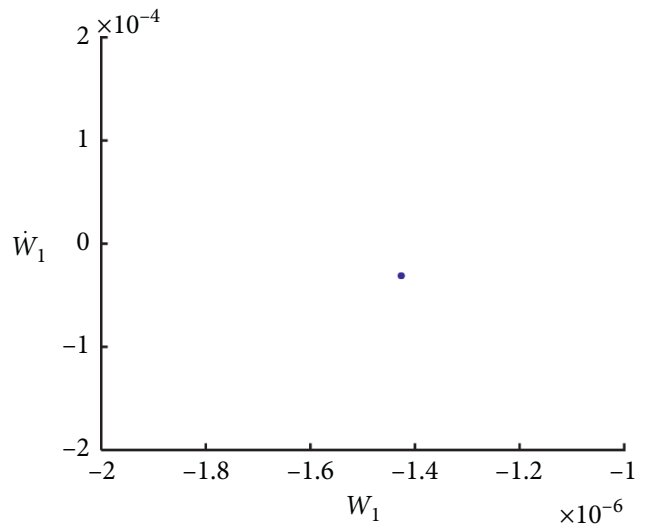

(f)

Figure 15: Periodic motion of the stiffened cylindrical shell when $N_{1}=6.5 \times 10^{7} \mathrm{~N} / \mathrm{m}^{2}$. (a) Phase portraits on the plane $\left(W_{1}, \dot{W}_{1}\right)$. (b) Waveform on the plane $\left(t, W_{1}\right)$. (c) Phase portraits on the plane. (d) Waveform on the plane $\left(t, W_{2}\right)$. (e) Three-dimensional phase portrait $\left(W_{1}, \dot{W}_{1}, W_{2}\right)$. (f) Poincare map on plane $\left(W_{1}, \dot{W}_{1}\right)$.

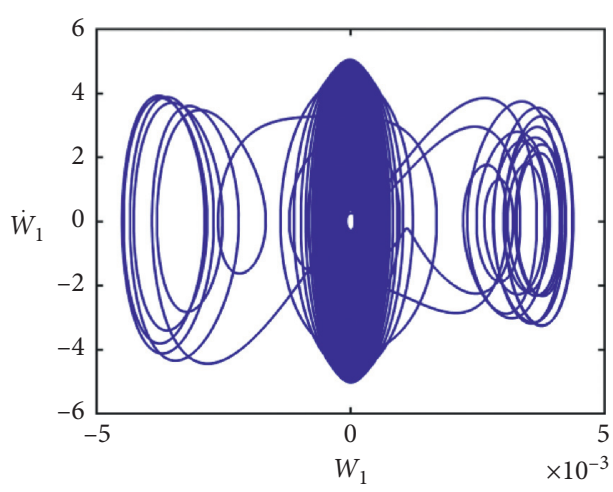

(a)

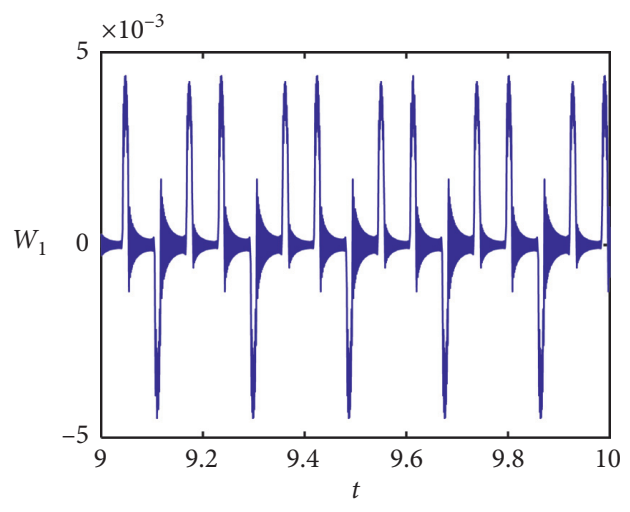

(b)

FIgURE 16: Continued. 


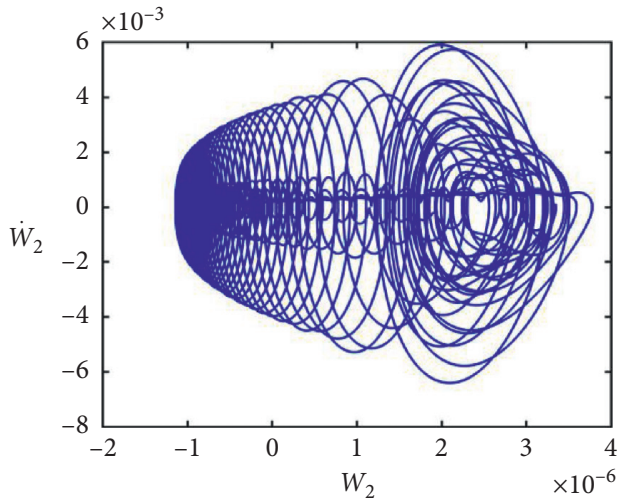

(c)

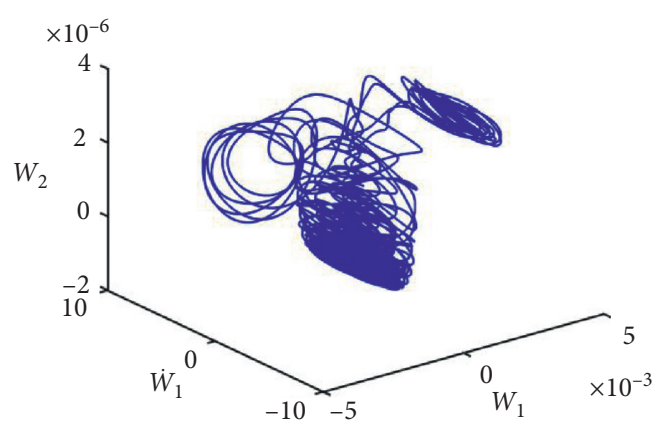

(e)

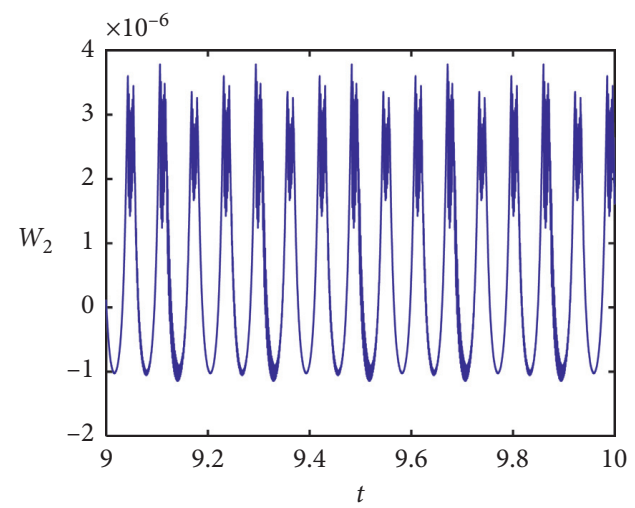

(d)

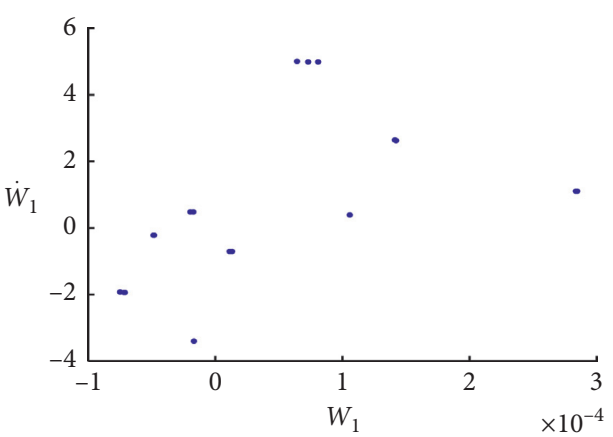

(f)

FIgURE 16: Chaotic motion of the stiffened cylindrical shell when $N_{1}=9.5 \times 10^{7} \mathrm{~N} / \mathrm{m}^{2}$. (a) Phase portraits on the plane $\left(W_{1}, \dot{W}_{1}\right)$. (b) Waveform on the plane $\left(t, W_{1}\right)$. (c) Phase portraits on the plane $\left(W_{2}, \dot{W}_{2}\right)$. (d) Waveform on the plane. (e) Three-dimensional phase portrait $\left(W_{1}, \dot{W}_{1}, W_{2}\right)$. (f) Poincare map on plane $\left(W_{1}, \dot{W}_{1}\right)$.

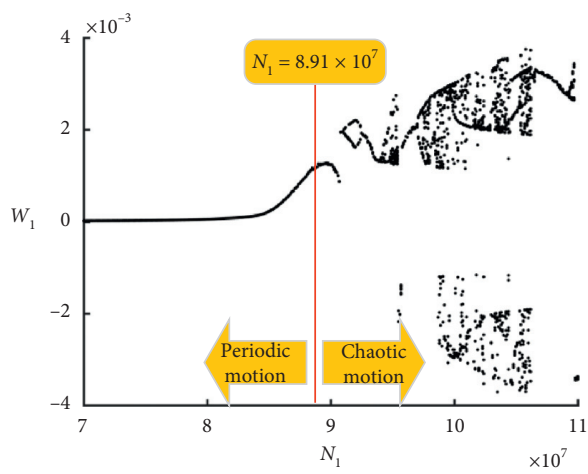

(a)

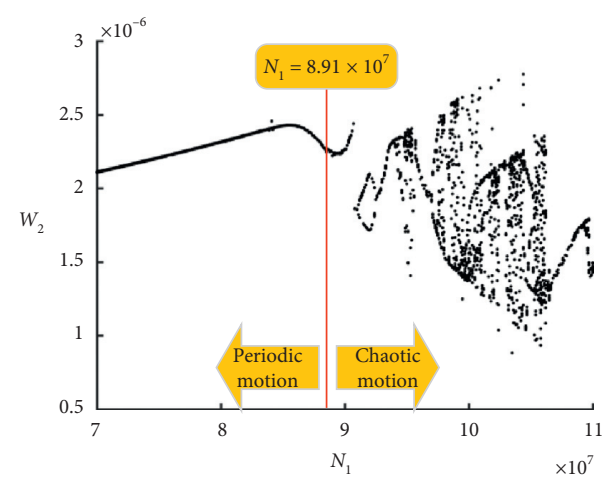

(b)

FiguRe 17: Bifurcation diagrams of stiffened cylindrical shell with length diameter ratio $L / R=3.5$. (a) First-order bifurcation diagram. (b) Second-order bifurcation diagram.

of the first two modes are described in Figure 17. As shown in Figure 17, the shell's motions translate periodic motions to chaotic motions when the critical value $N_{1}$ reaches $8.91 \times 10^{7} \mathrm{~N} / \mathrm{m}^{2}$. Moreover, the nonlinear dynamic responses of $N_{1}=7 \times 10^{7} \mathrm{~N} / \mathrm{m}^{2}$ and $10.19 \times 10^{7} \mathrm{~N} / \mathrm{m}^{2}$ are depicted in Figures 18 and 19, respectively. By comparing Figures 8 and 17, we find that, with the increase of aspect ratio from 2 to 3.5, the critical value of in-plane excitation $N_{1}$ increases from $5.92 \times 10^{7} \mathrm{~N} / \mathrm{m}^{2}$ to $9.37 \times 10^{7} \mathrm{~N} / \mathrm{m}^{2}$, which indicates that the larger aspect ratio can well prevent the chaotic phenomenon in the system.

Note that, in order to clearly explore the nonlinear behavior under various parameters, the representative parameters (i.e., stiffener number, stiffener height, and aspect ratio) are selected. Although the other stiffened cylindrical shells with different parameter combinations can also be 


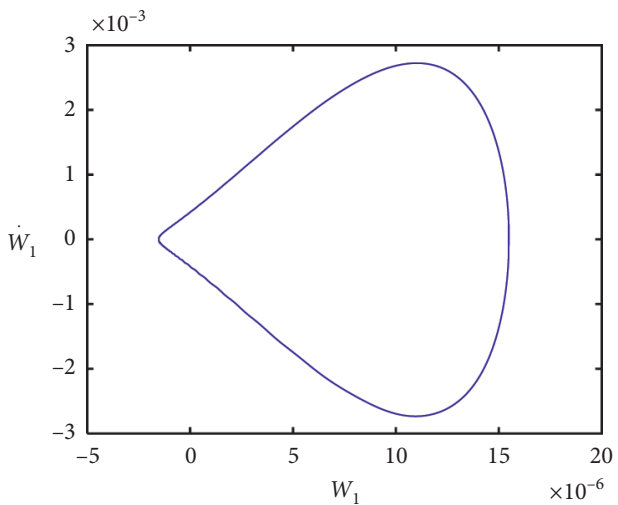

(a)

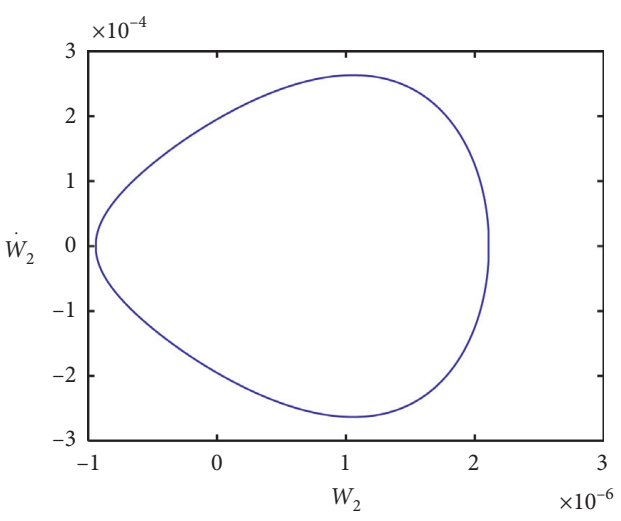

(c)

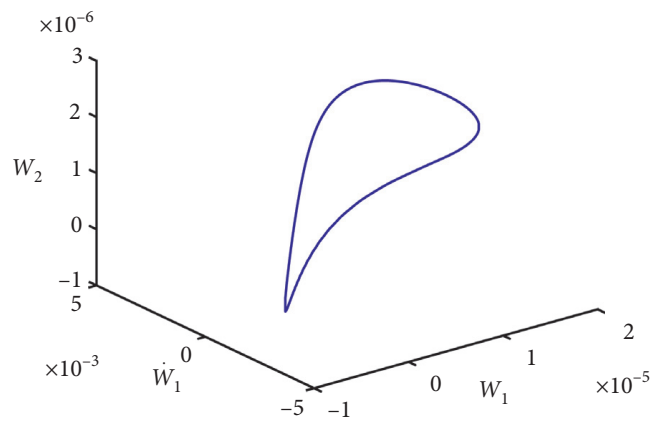

(e)

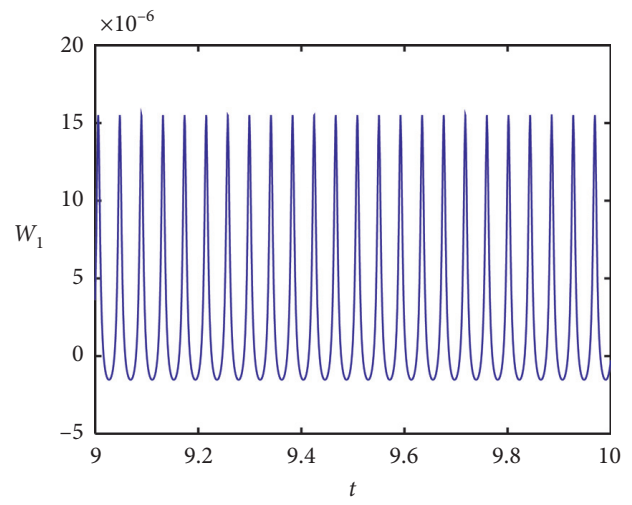

(b)

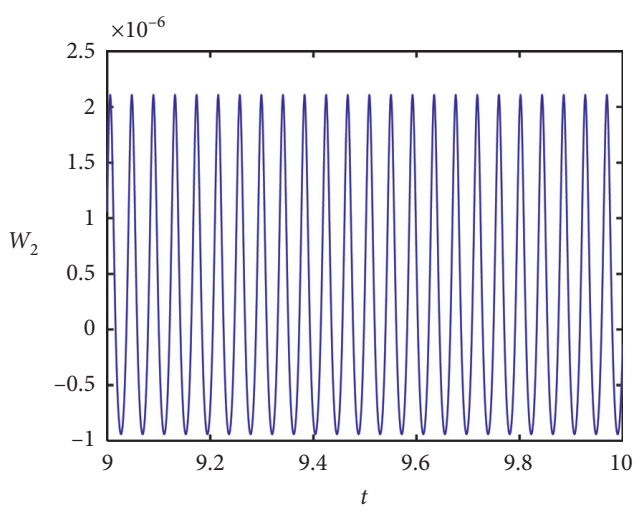

(d)

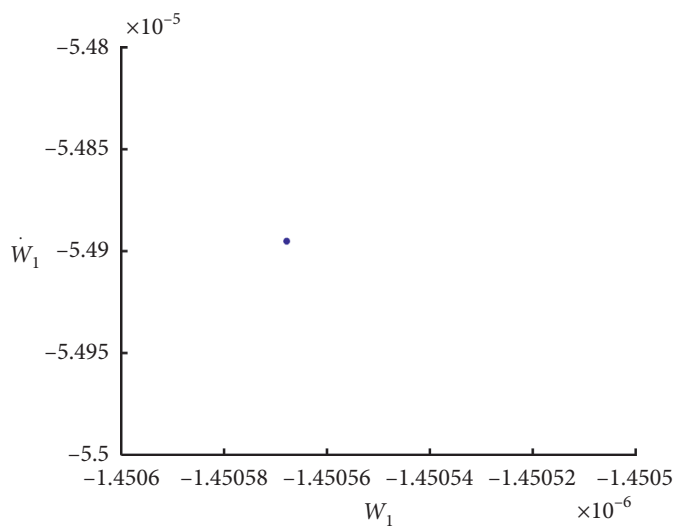

(f)

FIgURe 18: Periodic motion of the stiffened cylindrical shell when $N_{1}=7 \times 10^{7} \mathrm{~N} / \mathrm{m}^{2}$. (a) Phase portraits on the plane $\left(W_{1}, \dot{W}_{1}\right)$. $(\mathrm{b})$ Waveform on the plane $\left(t, W_{1}\right)$. (c) Phase portraits on the plane $\left(W_{2}, \dot{W}_{2}\right)$. (d) Waveform on the plane $\left(t, W_{2}\right)$. (e) Three-dimensional phase portrait $\left(W_{1}, \dot{W}_{1}, W_{2}\right)$. (f) Poincare map on plane $\left(W_{1}, \dot{W}_{1}\right)$. 


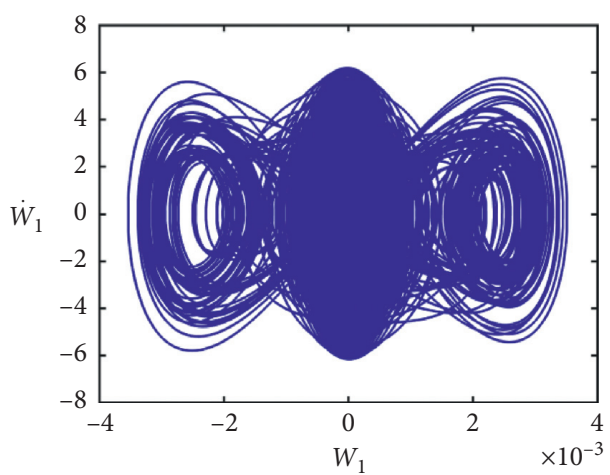

(a)

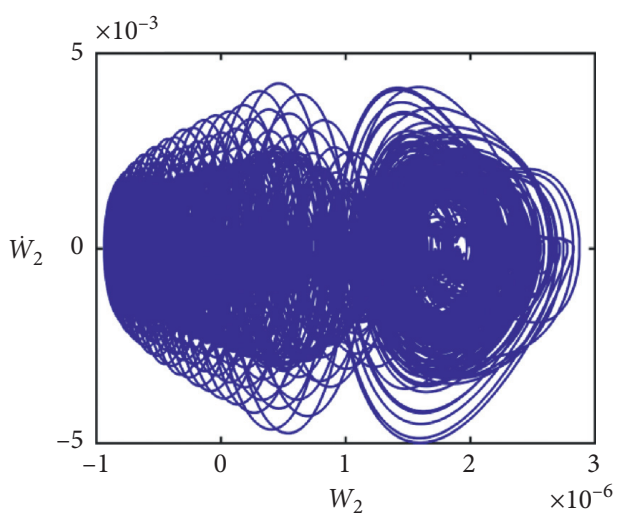

(c)

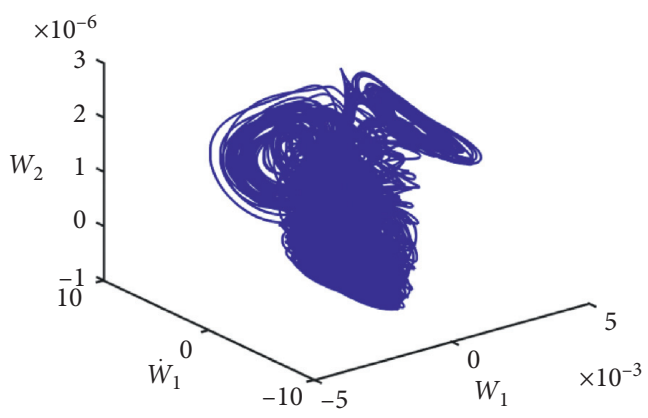

(e)

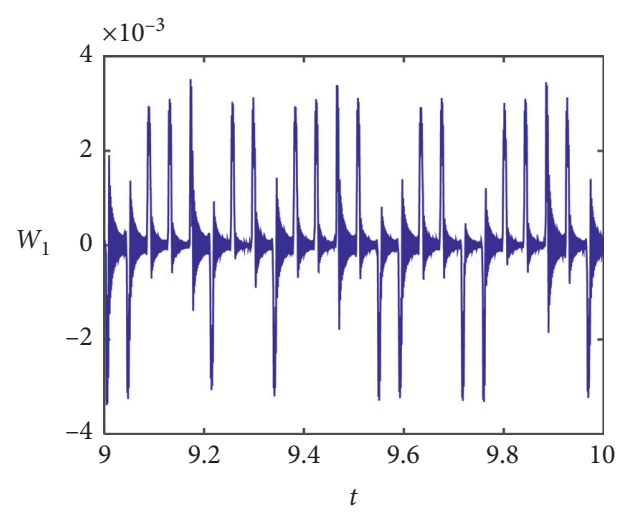

(b)

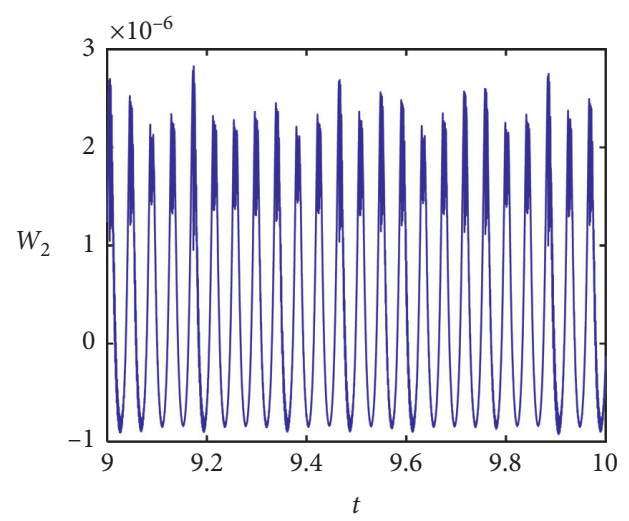

(d)

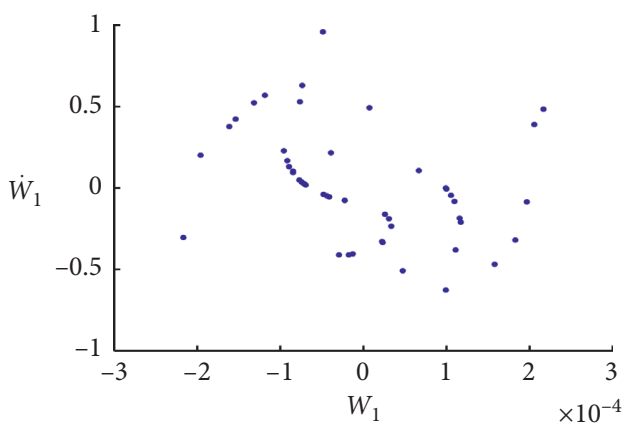

(f)

Figure 19: Chaotic motion of the stiffened cylindrical shell when $N_{1}=10.19 \times 10^{7} \mathrm{~N} / \mathrm{m}^{2}$. (a) Phase portraits on the plane $\left(W_{1}, \dot{W}_{1}\right)$. (b) Waveform on the plane $\left(t, W_{1}\right)$. (c) Phase portraits on the plane $\left(W_{2}, \dot{W}_{2}\right)$. (d) Waveform on the plane $\left(t, W_{2}\right)$. (e) Three-dimensional phase portrait $\left(W_{1}, \dot{W}_{1}, W_{2}\right)$. (f) Poincare map on plane $\left(W_{1}, \dot{W}_{1}\right)$.

calculated by adopting the proposed method, it should be explored in further investigations.

\section{Conclusions}

In this study, the nonlinear vibration analysis for stiffened cylindrical shells subjected to electromagnetic excitations, transverse excitations, and in-plane excitations is studied for the first time. Firstly, in the framework of the first-order shear deformation theory, the nonlinear partial differential governing equations of motion for stiffened cylindrical shells are derived by using Hamilton's principle. Then, the nonlinear partial differential governing equations of motion are diverted into a set of coupled nonlinear ordinary differential equations by the Galerkin discretization procedure. By using the multiscale method of perturbation analysis, we obtain four-dimensional nonlinear averaged equations in the polar forms under the case of $1: 2$ internal resonance and principal resonance-1/2 subharmonic parametric resonance. At last, we investigate the effects of different parameters on the resonant responses and the chaotic dynamics of the stiffened cylindrical shell. During the aforementioned analysis, the nonlinear vibrations of stiffened cylindrical shells can be concluded as follows: 
(1) From the analysis of resonant responses, we find that multiple amplitudes and jump phenomena have appeared in amplitude-frequency curves. By decreasing the external excitation or increasing the magnetic intensity, the unstable regions of stiffened cylindrical shells can be narrowed and the test accuracy of armature structures can be guaranteed effectively.

(2) With the numerical simulation of periodic and chaotic motions, the bifurcation diagrams, phase portraits, waveforms, and Poincare maps are acquired. From the simulation results, we discover that the periodic motions are translated to chaotic motions as the increase of in-plane excitation. Therefore, the working frequency range of armature structures can be expanded by reducing the in-plane excitation.

(3) With the influence investigations of geometric configurations on nonlinear dynamics, we notice that, with the increase of stiffener number, stiffener size, and aspect ratio, the chaotic phenomenon can be suppressed effectively and the high-accuracy vibration waveform can be produced successfully. Thus, the efforts are of great significance.

Since unstable nonlinear dynamic behaviors impose huge waveform deviations of armature structures, we can control multiple excitations (i.e., external excitation and magnetic intensity) and geometric configurations (i.e., stiffener number, stiffener size, and aspect ratio) in order to enhance the test accuracy of armature structures. The presented approach in this paper can provide an efficient analytical framework for nonlinear dynamics theories of stiffened cylindrical shells and will supply important technique guidance for armature structure design in vibration test engineering. Note that the analysis results of this paper are calculated by strict mathematical reasoning, which can effectively ensure its correctness. Other methods will be used to solve partial differential equations of dynamics equation (16), to further verify the proposed numerical model and its solutions.

\section{Data Availability}

All data included in this study are available from the corresponding author upon request.

\section{Conflicts of Interest}

The authors declare that there are no conflicts of interest regarding the publication of this paper.

\section{Acknowledgments}

This paper was co-supported by the National Natural Science Foundation of China (Grant 51975028), the China Postdoctoral Science Foundation (Grant 2021M690290), and the National Science and Technology Major Project (Grant J2019-IV-0016-0084). The authors would like to acknowledge them.

\section{References}

[1] J. Martino and K. Harri, "Virtual shaker modeling and simulation, parameters estimation of a high damped electrodynamic shaker," International Journal of Mechanical Sciences, vol. 151, pp. 375-384, 2019.

[2] M. S. Amjad, A. Rehman, M. Z. Amjad, and M. Usman, "Structural dynamics modification of vibration-test fixture," Journal of Testing and Evaluation, vol. 45, no. 6, pp. 20832092, 2017.

[3] D. Ramkrishna, A. Joshi, P. M. Mujumdar, and Y. Krishna, "Frequency domain input identification for vibration testing of flexible structures," Proceedings of the Institution of $\mathrm{Me}$ chanical Engineers-Part G: Journal of Aerospace Engineering, vol. 227, no. G5, pp. 838-856, 2013.

[4] L. Z. Zhou, J. L. Han, and G. Y. Ji, "Optimum design of new high strength electrodynamic shaker in broad work frequency range," Journal of Vibroengineering, vol. 16, no. 5, pp. 25522562, 2014.

[5] H. Dal and M. Baklac1, "Design, fabrication and vibration analysis of a lightweight head expander for a high frequency electrodynamic shaker," Materials Testing, vol. 61, no. 10, pp. 965-972, 2019.

[6] A. Swaminathan, M. E. Poese, R. W. M. Smith, and S. L. Garrett, "Note: a kinematic shaker system for high amplitude, low frequency vibration testing," Review of Scientific Instruments, vol. 86, no. 11, Article ID 116102, 2015.

[7] M. Saadatzi, M. N. Saadatzi, V. Tavaf, and S. Banerjee, "Aeve 3D: acousto electrodynamic three-dimensional vibration exciter for engineering testing," IEEE, vol. 23, no. 4, pp. 1897-1906, 2018.

[8] M. Ayub, G. J. Sirewal, S. S. H. Bukhari, and B. I. Kwon, "Brushless wound rotor synchronous machine with thirdharmonic field excitation," Electrical Engineering, vol. 102, no. 1SI, pp. 259-265, 2020.

[9] L.-K. Song, G.-C. Bai, and X.-Q. Li, "A novel metamodeling approach for probabilistic LCF estimation of turbine disk," Engineering Failure Analysis, vol. 120, Article ID 105074, 2021.

[10] C. Lu, C.-W. Fei, Y.-W. Feng, Y.-J. Zhao, X.-W. Dong, and Y.-S. Choy, "Probabilistic analyses of structural dynamic response with modified Kriging-based moving extremum framework," Engineering Failure Analysis, vol. 125, Article ID 105398, 2021.

[11] X.-Q. Li, G.-C. Bai, L.-K. Song, and J. Wen, "Fatigue reliability estimation framework for turbine rotor using multi-agent collaborative modeling," Structure, vol. 29, pp. 1967-1978, 2021.

[12] L. Han, C. Chen, T. Guo et al., "Probability-based service safety life prediction approach of raw and treated turbine blades regarding combined cycle fatigue," Aerospace Science and Technology, vol. 110, Article ID 106513, 2021.

[13] C. W. Fei, H. T. Liu, S. L. Li, H. Li, L. Q. An, and C. Lu, "Dynamic parametric modeling-based model updating strategy of aeroengine casings," Chinese Journal of Aeronautics, 2021, In press.

[14] B. Keshtegar, M. Bagheri, C. W. Fei, C. Lu, O. Taylan, and D. K. Thai, "Multi-extremum modified response basis model for nonlinear response prediction of dynamic turbine blisk," Engineering with Computers, 2021, In press.

[15] T. Liu, W. Zhang, J. J. Mao, and Y. Zheng, "Nonlinear breathing vibrations of eccentric rotating composite laminated circular cylindrical shell subjected to temperature, rotating speed and external excitations," Mechanical Systems and Signal Processing, vol. 127, pp. 463-498, 2019. 
[16] P. Oliazadeh and A. Farshidianfar, "Analysis of different techniques to improve sound transmission loss in cylindrical shells," Journal of Sound and Vibration, vol. 389, pp. 276-291, 2017.

[17] M. S. Boroujerdy and M. R. Eslami, "Nonlinear axisymmetric thermomechanical response of piezo-FGM shallow spherical shells," Archive of Applied Mechanics, vol. 83, no. 12, pp. 1681-1693, 2013.

[18] R. Ramzi and A. Lakis, "Effect of geometric nonlinearities on nonlinear vibrations of closed cylindrical shells," International Journal of Structural Stability and Dynamics, vol. 13, no. 4, Article ID 1250078, 2013.

[19] H. Farokhi and M. H. Ghayesh, "Nonlinear mechanical behaviour of microshells," International Journal of Engineering Science, vol. 127, pp. 127-144, 2018.

[20] M. Amabili and P. Balasubramanian, "Nonlinear vibrations of truncated conical shells considering multiple internal resonances," Nonlinear Dynamics, vol. 100, no. 1, pp. 77-93, 2020.

[21] M. Strozzi, V. V. Smirnov, L. I. Manevitch, and F. Pellicano, "Nonlinear normal modes, resonances and energy exchange in single-walled carbon nanotubes," International Journal of Non-linear Mechanics, vol. 120, Article ID 103398, 2020.

[22] S. Anicic, "Existence theorem for a first-order Koiter nonlinear shell model," Discrete \& Continuous Dynamical Systems-Series S, vol. 12, no. 6, pp. 1535-1545, 2019.

[23] D. G. Ninh, N. D. Tien, V. N. V. Hoang, and D. H. Bich, "Vibration of cylindrical shells made of three layers W-Cu composite containing heavy water using Flügge-Lur'e-Bryrne theory," Thin-Walled Structures, vol. 146, Article ID 106414, 2020.

[24] N. D. Tien, V. N. V. Hoang, D. G. Ninh, V. L. Huy, and N. C. Hung, "Nonlinear dynamics and chaos of a nanocomposite plate subjected to electro-thermo-mechanical loads using Flugge-Lur'e-Bryrne theory," Journal of Vibration and Control, vol. 27, 2020.

[25] H. A. Zamani, "Nonlinear vibration of piezoelectric graphenereinforced composite laminated panels in thermal environment using Amabili-Reddy shear deformation theory," Composite Structures, vol. 250, Article ID 112556, 2020.

[26] W. Zhang, Z. Fang, X.-D. Yang, and F. Liang, "A series solution for free vibration of moderately thick cylindrical shell with general boundary conditions," Engineering Structures, vol. 165, pp. 422-440, 2018.

[27] X. Q. Li, W. Zhang, X. D. Yang, and L. K. Song, “A unified approach of free vibration analysis for stiffened cylindrical shell with general boundary conditions," Mathematical Problems in Engineering, vol. 2019, Article ID 4157930, 14 pages, 2019.

[28] T. Liu, W. Zhang, and J. F. Wang, "Nonlinear dynamics of composite laminated circular cylindrical shell clamped along a generatrix and with membranes at both ends," Nonlinear Dynamics, vol. 90, no. 2, pp. 1393-1417, 2017.

[29] Y. Gholami, R. Ansari, R. Gholami, and H. Rouhi, “Analyzing primary resonant dynamics of functionally graded nanoplates based on a surface third-order shear deformation model," Thin-Walled Structures, vol. 131, pp. 487-499, 2018.

[30] W. Zhang, T. Liu, A. Xi, and Y. N. Wang, "Resonant responses and chaotic dynamics of composite laminated circular cylindrical shell with membranes," Journal of Sound and Vibration, vol. 423, pp. 65-99, 2018.

[31] M. Amabili, "Nonlinear vibrations and stability of laminated shells using a modified first-order shear deformation theory," European Journal of Mechanics-A: Solids, vol. 68, pp. 75-87, 2018.
[32] A. A. Yazdi, "Nonlinear vibration of thick multi-scale composite doubly curved shells," Mechanics Based Design of Structures and Machines, Article ID 1760882, 2020.

[33] M. Karimiasl, F. Ebrahimi, and M. Vinyas, "Nonlinear vibration analysis of multiscale doubly curved piezoelectric composite shell in hygrothermal environment," Journal of Intelligent Material Systems and Structures, vol. 30, no. 10, pp. 1594-1609, 2019.

[34] X. J. Gu, Y. X. Hao, W. Zhang, and J. Chen, "Dynamic stability of rotating cantilever composite thin walled twisted plate with initial geometric imperfection under in-plane load," ThinWalled Structures, vol. 144, Article ID 106267, 2019.

[35] M. Amabili and J. N. Reddy, "A new non-linear higher-order shear deformation theory for large-amplitude vibrations of laminated doubly curved shells," International Journal of Non-linear Mechanics, vol. 45, no. 4, pp. 409-418, 2010.

[36] Y. Zheng, W. Zhang, and T. Liu, "Coexistence of doubleparameter nonlinear dynamics and metastable chaos for bistable asymmetric composite laminated square panel under combined external and parametric excitations," Nonlinear Dynamics, vol. 104. , 2021 In press.

[37] W. Zhang, Y. Zheng, T. Liu, and X. Y. Guo, "Multi-pulse jumping double-parameter chaotic dynamics of eccentric rotating ring truss antenna under combined parametric and external excitations," Nonlinear Dynamics, vol. 98, no. 1, pp. 761-800, 2019.

[38] Y. F. Zhang, T. Liu, and W. Zhang, "Nonlinear resonant responses, mode interactions, and multitime periodic and chaotic oscillations of a cantilevered pipe conveying pulsating fluid under external harmonic force," Complexity, vol. 2020, Article ID 9840860, 26 pages, 2020.

[39] P. B. Gonçalves and Z. G. del Prado, "Effect of non-linear modal interaction on the dynamic instability of axially excited cylindrical shells," Computers \& Structures, vol. 82, no. 31-32, pp. 2621-2634, 2004.

[40] T. Liu, W. Zhang, Y. Zheng, and Y. F. Zhang, "AndronovHopf bifurcations, Pomeau-Manneville intermittent chaos and nonlinear vibrations of large deployable space antenna subjected to thermal load and radial pre-stretched membranes with 1:3 internal resonance," Chaos, Solitons \& Fractals, vol. 144, Article ID 110719, 2021.

[41] V. Dogan and R. Vaicaitis, "Nonlinear response of doublewall cylindrical shell vibrations under random excitation," Journal of Aerospace Engineering, vol. 19, no. 1, pp. 46-54, 2006.

[42] A. Kumar and B. P. Patel, "Nonlinear dynamic response of elliptical cylindrical shell underHarmonic excitation," International Journal of Non-Linear Mechanics, vol. 98, pp. 102113, 2018.

[43] N. Dinh Duc and P. T. Thang, "Nonlinear response of imperfect eccentrically stiffened ceramic-metal-ceramic sigmoid functionally graded material (S-FGM) thin circular cylindrical shells surrounded on elastic foundations under uniform radial load," Mechanics of Advanced Materials and Structures, vol. 22, no. 12, pp. 1031-1038, 2015.

[44] I. D. Breslavsky and M. Amabili, "Nonlinear vibrations of a circular cylindrical shell with multiple internal resonances under multi-harmonic excitation," Nonlinear Dynamics, vol. 93, no. 1, pp. 53-62, 2018.

[45] P. Edalata, M. R. Khedmati, and C. G. Soares, "Free vibration and dynamic response analysis of stiffened parabolic shells using equivalent orthotropic shell parameters," Latin American Journal of Solids and Structures, vol. 10, no. 4, pp. 747766, 2013. 
[46] C. Pan, X. Sun, and Y. Zhang, "Vibro-acoustic analysis of submerged ring-stiffened cylindrical shells based on a symplectic wave-based method," Thin-Walled Structures, vol. 150, Article ID 106698, 2020.

[47] K. Foroutan, A. Shaterzadeh, and H. Ahmadi, "Nonlinear dynamic analysis of spiral stiffened cylindrical shells rested on elastic foundation," Steel and Composite Structures, vol. 32, no. 4, pp. 509-519, 2019.

[48] M. Valentin, M. Laurent, R. Ygaal, and A. Christian, "Vibroacoustic modelling of submerged stiffened cylindrical shells with internal structures under random excitations," in Proceedings of the InterNoise16 (2016), pp. 2390-2401, Institute of Noise Control Engineering, Hamburg, Germany, August 2016.

[49] M. Noroozi and F. Bakhtiari-Nejad, "Nonlinear vibration of a nanocomposite laminated piezoelectric trapezoidal actuator in subsonic airflow under combined electrical and forcing excitations," Proceedings of the Institution of Mechanical Engineers-Part C: Journal of Mechanical Engineering Science, 2020.

[50] M. Mir and M. Tahani, "Graphene-based mass sensors: chaotic dynamics analysis using the nonlocal strain gradient model," Applied Mathematical Modelling, vol. 81, pp. 799$817,2020$.

[51] M. Mansuripur, "Nature of electric and magnetic dipoles gleaned from the poynting theorem and the Lorentz force law of classical electrodynamics," Optics Communications, vol. 284, no. 2, pp. 594-602, 2011.

[52] I. Galili, D. Kaplan, and Y. Lehavi, “Teaching Faraday's law of electromagnetic induction in an introductory physics course," American Journal of Physics, vol. 74, no. 4, pp. 337-343, 2006.

[53] E. Dowell, "Hamilton's principle and Hamilton's equations with holonomic and non-holonomic constraints," Nonlinear Dynamics, vol. 88, no. 2, pp. 1093-1097, 2017.

[54] A. Nosier and J. N. Reddy, "A study of non-linear dynamic equations of higher-order shear deformation plate theories," International Journal of Non-linear Mechanics, vol. 26, no. 2, pp. 233-249, 1991.

[55] W. Zhang, R. Q. Wu, and K. Behdinan, "Nonlinear dynamic analysis near resonance of a beam-ring structure for modeling circular truss antenna under time-dependent thermal excitation," Aerospace Science and Technology, vol. 86, pp. 296311, 2019.

[56] J. E. Chen, W. Zhang, X. Y. Guo, and M. Sun, “Theoretical and experimental studies on nonlinear oscillations of symmetric cross-ply composite laminated plates," Nonlinear Dynamics, vol. 73, no. 3, pp. 1697-1714, 2013.

[57] Y. X. Hao, W. Zhang, S. B. Li, and J. H. Zhang, "Nonlinear vibrations of FGM cylindrical panel with simply supported edges in air flow," International Journal of Aerospace Engineering, vol. 2015, Article ID 246352, 14 pages, 2015.

[58] T. Liu, W. Zhang, M. Q. Wu, Y. Zheng, and Y. F. Zhang, "Metastable nonlinear vibrations: third chaos of bistable asymmetric composite laminated square shallow shell under foundation excitation," Composite Structures, vol. 255, Article ID 112966, 2021. 